\title{
Experimental Study of Single Spin Asymmetries and TMDs
}

\author{
Jian-ping Chen, Jefferson Lab
}

QCD Evolution Workshop, JLab, May 6-10, 2013

- Recent SSA Results from JLab Hall A with a Transversely Polarized ${ }^{3} \mathrm{He}(\mathrm{n})$

Collins/Sivers Asymmetries on pi+/pi- (published)

Worm-gear II Asymmetries on pi+/pi- (published)

- New Preliminary SSA Results from JLab Hall A with a Transversely polarized ${ }^{3} \mathrm{He}(\mathrm{n})$

Collins/Sivers Asymmetries in $\mathrm{K}+/ \mathrm{K}$ -

Pretzelosity Asymmetries on $\pi+l \pi$ -

Inclusive hadron SSA

Inclusive electron SSA (DIS, QE)

- TMD study at JLab $12 \mathrm{GeV}$ in Hall A:

SoLID Program on SSA/TMDs: 3 Approved Experiments on ${ }^{3} \mathrm{He}$ and $p$

New LOI on dihadron production

- Long-term Future: TMDs study with Electron-Ion Colliders (EIC)

MEIC@JLab and E-RHIC@BNL

A New Opportunity: an EIC in China (EIC@HIAF) 


\title{
Single Spin Asymmetries with A Transversely Polarized ${ }^{3} \mathrm{He}(\mathrm{n})$
}

\author{
JLab Hall A E06-010
}




\section{Leading-Twist TMD PDFs}

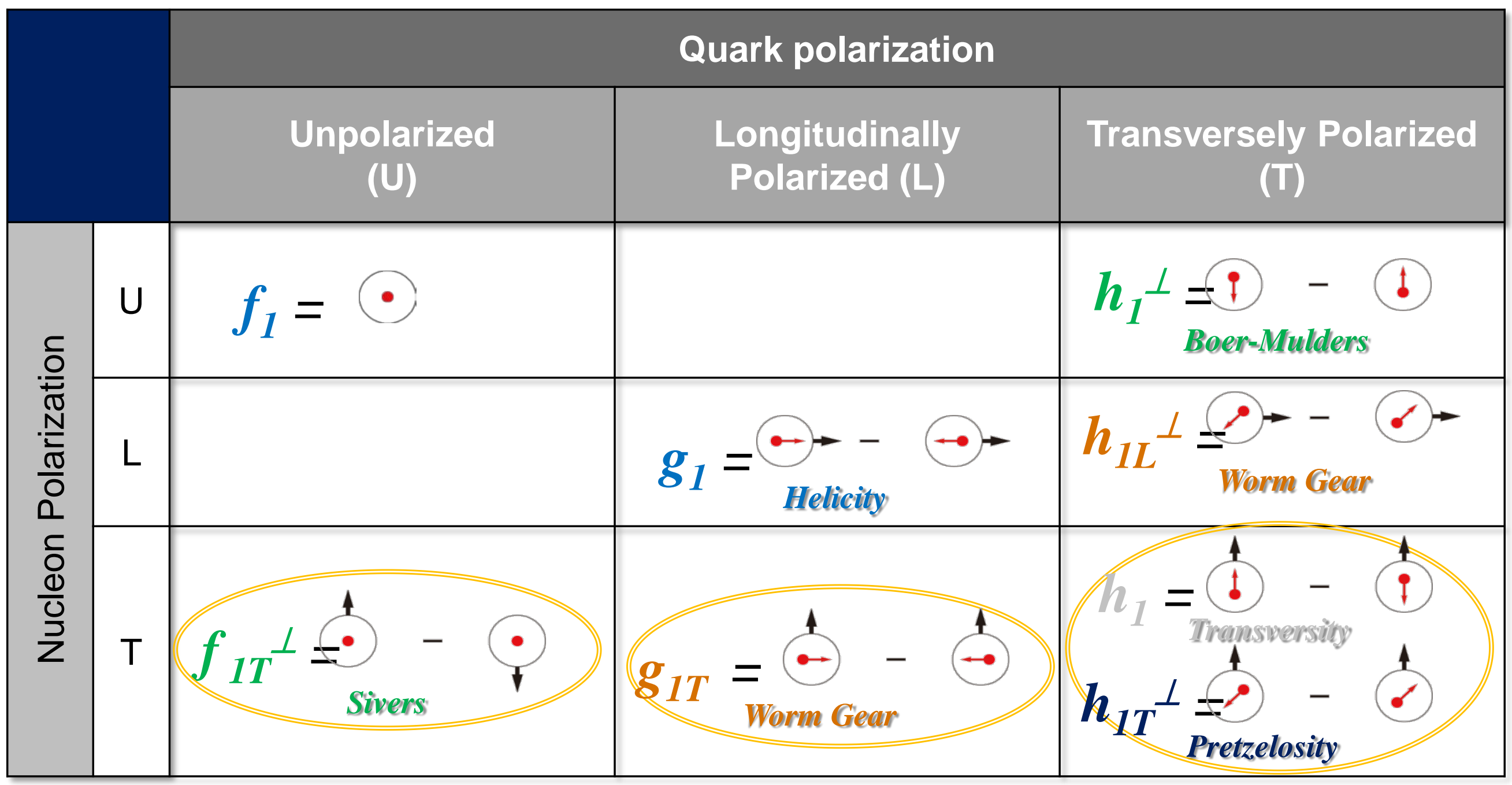
$\rightarrow$ Nucleon Spin
$\Leftrightarrow$ Quark Spin
" Probed with transversely pol target HERMES, COMPASS, JLab E06-010 


\section{Separation of Collins, Sivers and pretzelocity effects through angular dependence}

$$
\begin{aligned}
& A_{U T}\left(\varphi_{h}^{l}, \varphi_{S}^{l}\right)=\frac{1}{P} \frac{N^{\uparrow}-N^{\downarrow}}{N^{\uparrow}+N^{\downarrow}} \\
& =A_{U T}^{\text {Collins }} \sin \left(\phi_{h}+\phi_{S}\right)+A_{U T}^{\text {Sivers }} \sin \left(\phi_{h}-\phi_{S}\right) \\
& +A_{U T}^{\text {Pretzelosity }} \sin \left(3 \phi_{h}-\phi_{S}\right)
\end{aligned}
$$

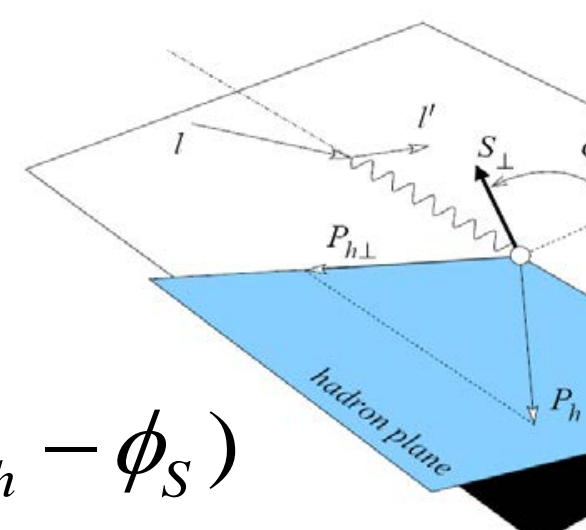

$A_{U T}^{\text {Collins }} \propto\left\langle\sin \left(\phi_{h}+\phi_{S}\right)\right\rangle_{U T} \propto h_{1} \otimes H_{1}^{\perp}$

$A_{U T}^{\text {Sivers }} \propto\left\langle\sin \left(\phi_{h}-\phi_{S}\right)\right\rangle_{U T} \propto f_{1 T}^{\perp} \otimes D_{1}$

$A_{U T}^{\text {Pretzelosity }} \propto\left\langle\sin \left(3 \phi_{h}-\phi_{S}\right)\right\rangle_{U T} \propto h_{1 T}^{\perp} \otimes H_{1}^{\perp}$ 


\section{E06-010 Experiment}

${ }^{3} H e^{\uparrow}\left(\vec{e}, e^{\prime} \pi^{ \pm}\right) X$

Spokespersons: Chen/Evaristo/Gao/Jiang/Peng ${ }^{3} H e^{\uparrow}\left(\vec{e}, e^{\prime} K^{ \pm}\right) X$

- First measurement on $n\left({ }^{3} \mathrm{He}\right)$

- Polarized ${ }^{3} \mathrm{He}$ Target

- Polarized Electron Beam, $5.9 \mathrm{GeV}$

- BigBite at 30 o as Electron Arm

$$
-P_{e}=0.7 \sim 2.2 \mathrm{GeV} / \mathrm{c}
$$

- $\mathrm{HRS}_{\mathrm{L}}$ at 160 as Hadron Arm

$-\boldsymbol{P}_{\boldsymbol{h}}=2.35 \mathrm{GeV} / \mathrm{c}$

- Excellent PID for $\pi / K / p$

- 7 PhD Thesis Students (All graduated) + new students

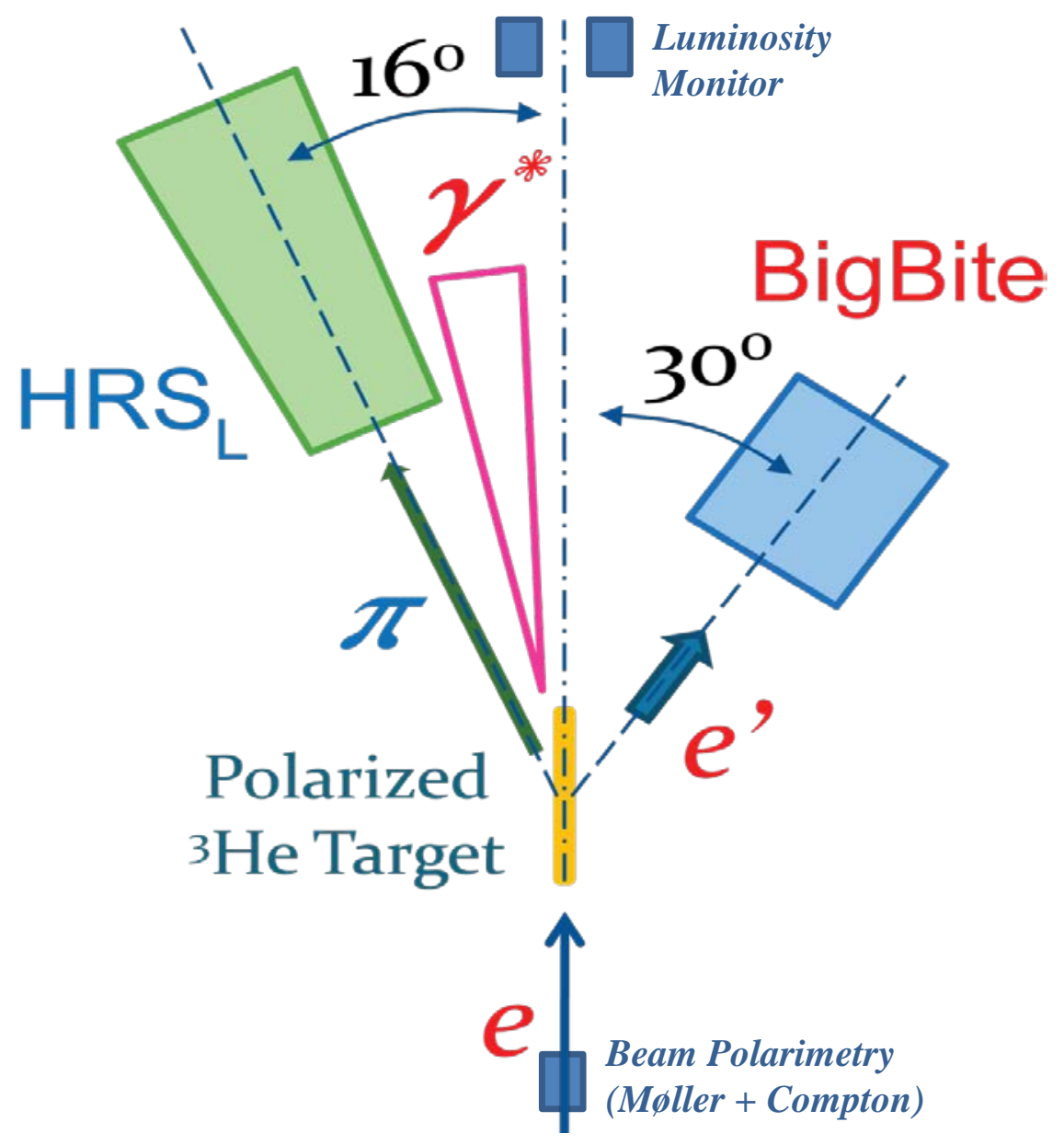




\section{Published Results (I) from JLab Hall A E06-010 with a Transversely Polarized ${ }^{3} \mathrm{He}(\mathrm{n})$}

Collins/Sivers Asymmetries on $\pi+/ \pi$ -

X. Qian at al., PRL 107:072003(2011) 


\section{E06-010 ${ }^{3} \mathrm{He}$ Target Single-Spin Asymmetry in SIDIS}

${ }^{3} \operatorname{He}^{\uparrow}\left(e, e^{\prime} h\right), h=\pi^{+}, \pi^{-}$

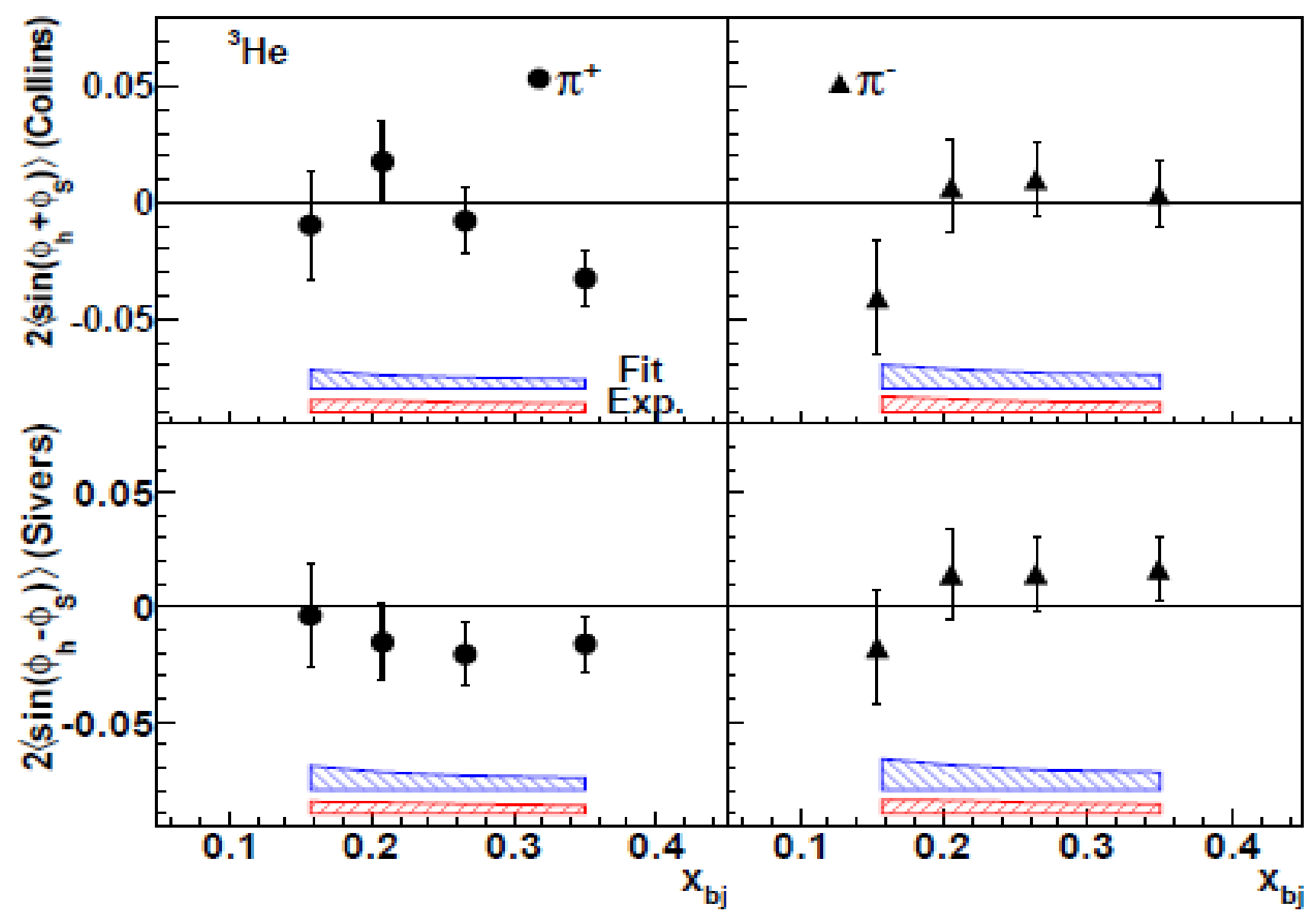

3He Collins SSA small

Non-zero at highest $\mathrm{x}$ for $\pi+$

Blue band: model (fitting) uncertainties

Red band: other systematic uncertainties

3He Sivers SSA:

negative for $\pi^{+}$ 


\section{Neutron Results with Polarized ${ }^{3} \mathrm{He}$ from JLab}

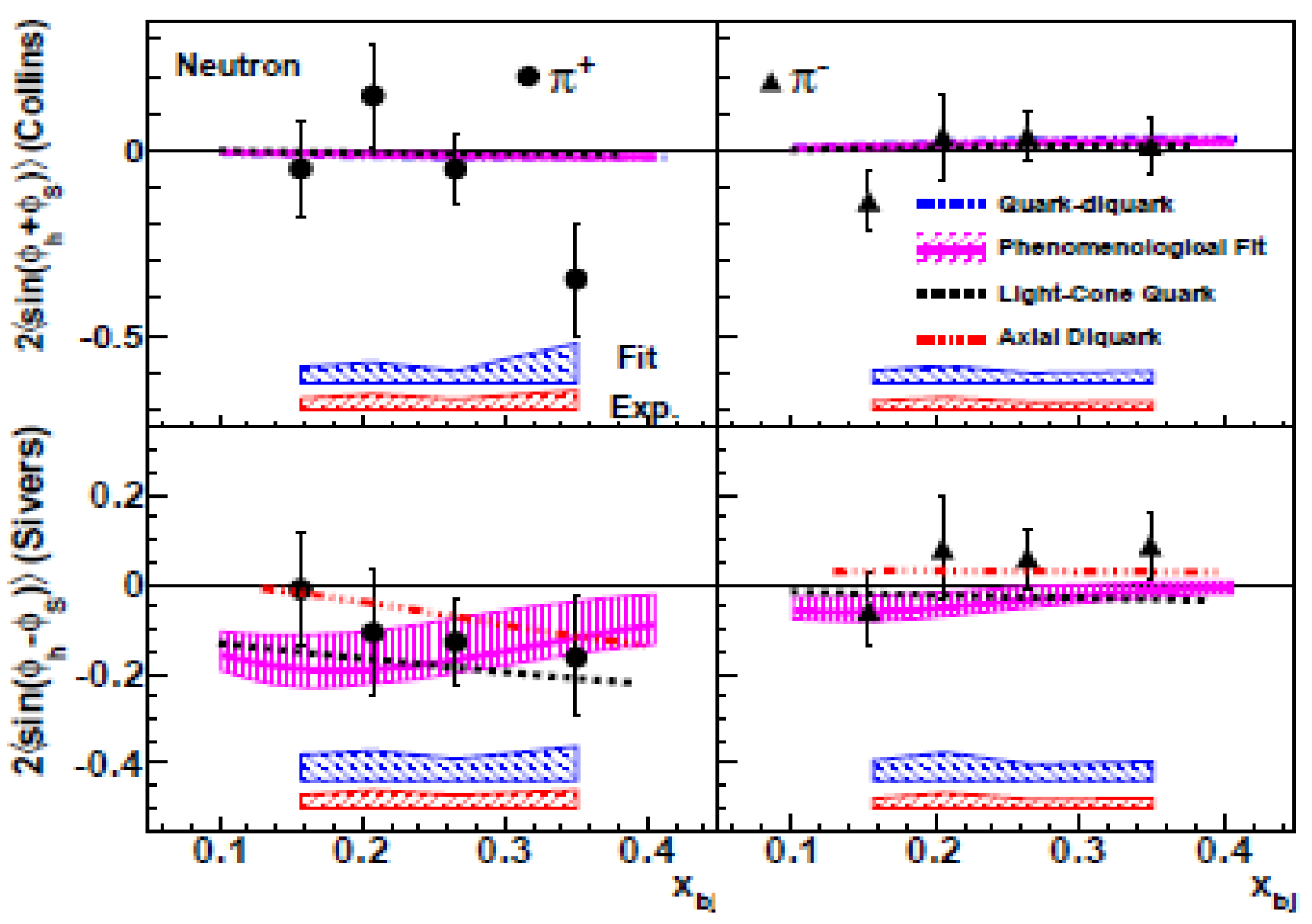

\section{Collins}

asymmetries are not large, except at $\mathrm{x}=0.34$

\section{Sivers}

$\pi^{+}(u \bar{d})$ negative

Blue band: model (fitting) uncertainties

Red band: other systematic uncertainties 


\title{
Published Results (II) from JLab Hall A E06-010 with a Transversely Polarized ${ }^{3} \mathrm{He}(\mathrm{n})$
}

\section{Worm-Gear II: Trans-helicity on $\pi+/ \pi-$}

\author{
J. Huang et al., PRL. 108, 052001 (2012).
}




\section{Asymmetry $A_{L T}$ Result}

To leading twist:

$$
A_{\mathrm{LT}}^{\cos \left(\phi_{h}-\phi_{s}\right)} \propto F_{L T}^{\cos \left(\phi_{h}-\phi_{s}\right)} \propto g_{1 T}^{q} \otimes D_{1 q}^{h}
$$

- ${ }^{3} \mathrm{He} A_{\mathrm{LT}}: \quad$ Positive for $\pi-$

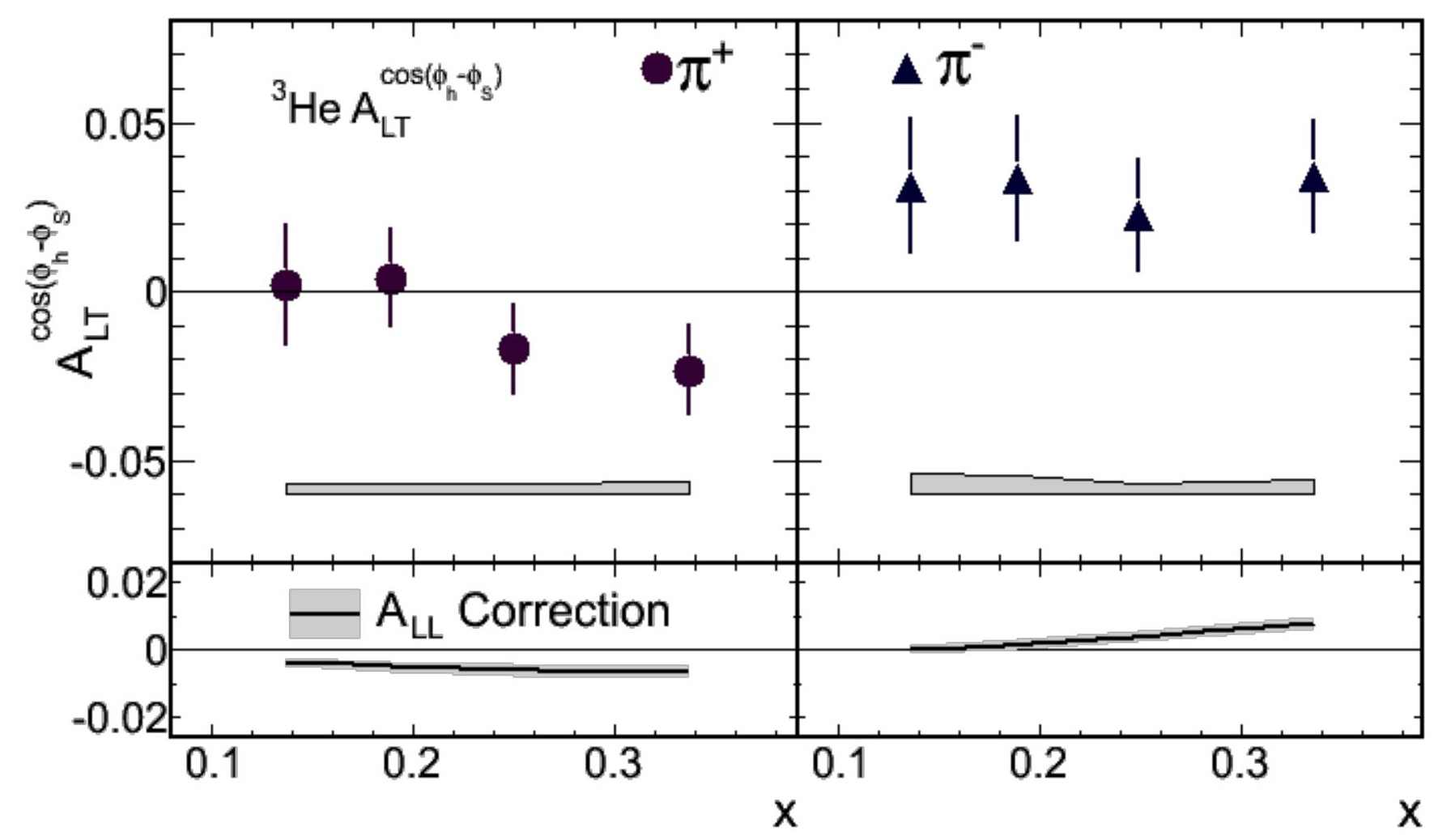




\section{Neutron $A_{L T}$ Extraction}

- $\quad A_{L T}^{3} \mathrm{He}=P_{n} \frac{\sigma_{n}}{\sigma_{3} \mathrm{He}} A_{L T}^{n}+P_{p} \frac{2 \sigma_{p}}{\sigma_{3} \mathrm{He}} A_{L T}^{p} \quad\left\{\begin{array}{l}P_{n}=0.86_{-0.02}^{+0.036} \\ P_{p}=-0.028_{-0.004}^{+0.009}\end{array}\right.$

- Corrected for proton dilution, $f_{p}$

- Predicted proton asymmetry contribution $<1.5 \%\left(\pi^{+}\right), 0.6 \%\left(\pi^{-}\right)$

- $A_{\mathrm{LT}}^{n} \propto g_{1 T}^{q} \otimes D_{1 q}^{h}$

- Dominated by $L=0(S)$ and $L=1(P)$ interference

- Consist w/ model in signs, suggest larger asymmetry

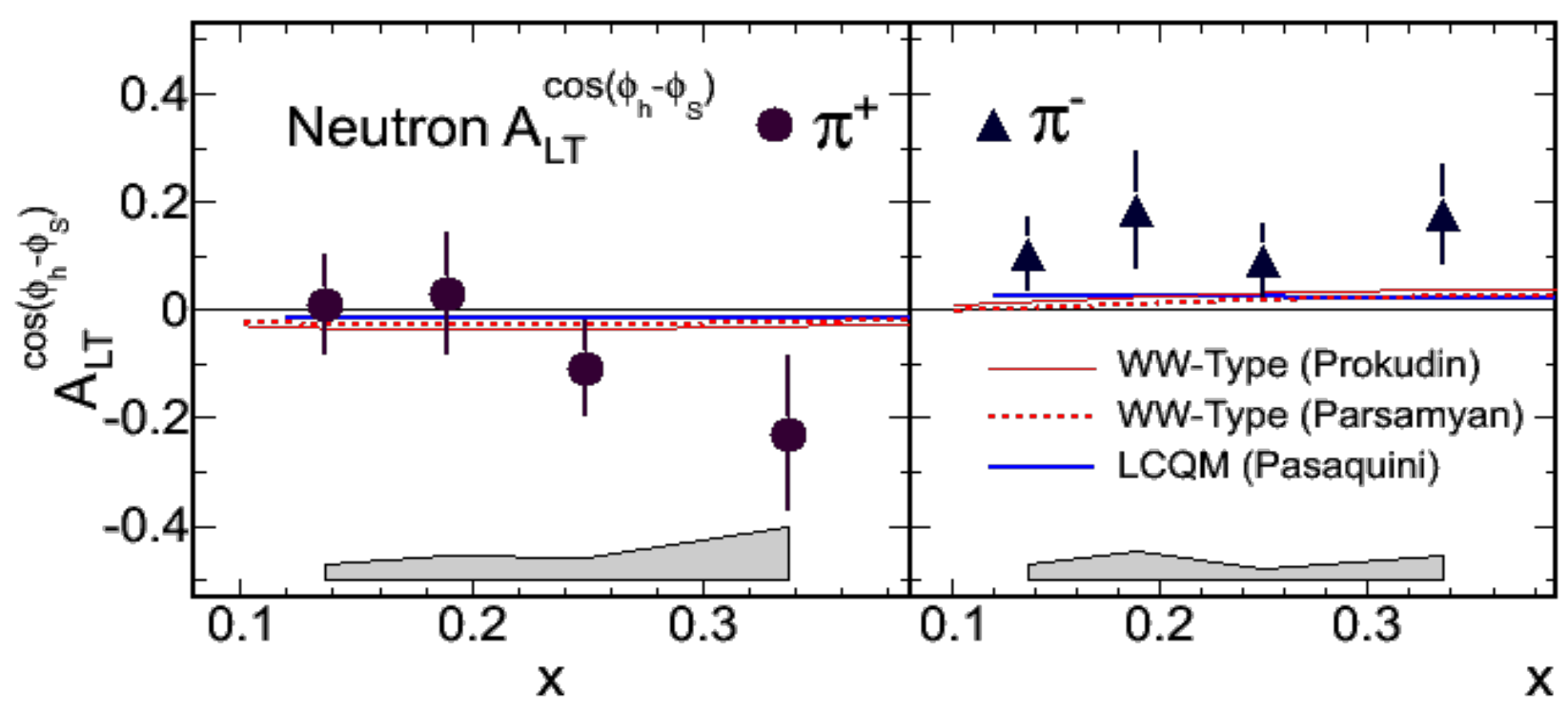




\section{Preliminary New Results (I) from JLab Hall A E06-010 with a Transversely Polarized ${ }^{3} \mathrm{He}$ (n)}

Collins/Sivers Asymmetries on $\mathrm{K}+/ \mathrm{K}-$

Analysis by Y. Zhao (USTC), Y. Wang (UIUC) 


\section{Kaon PID by Coincidence time of flight}

Cross checked with $\mathrm{RICH}$ results

CT.K.t for positive run

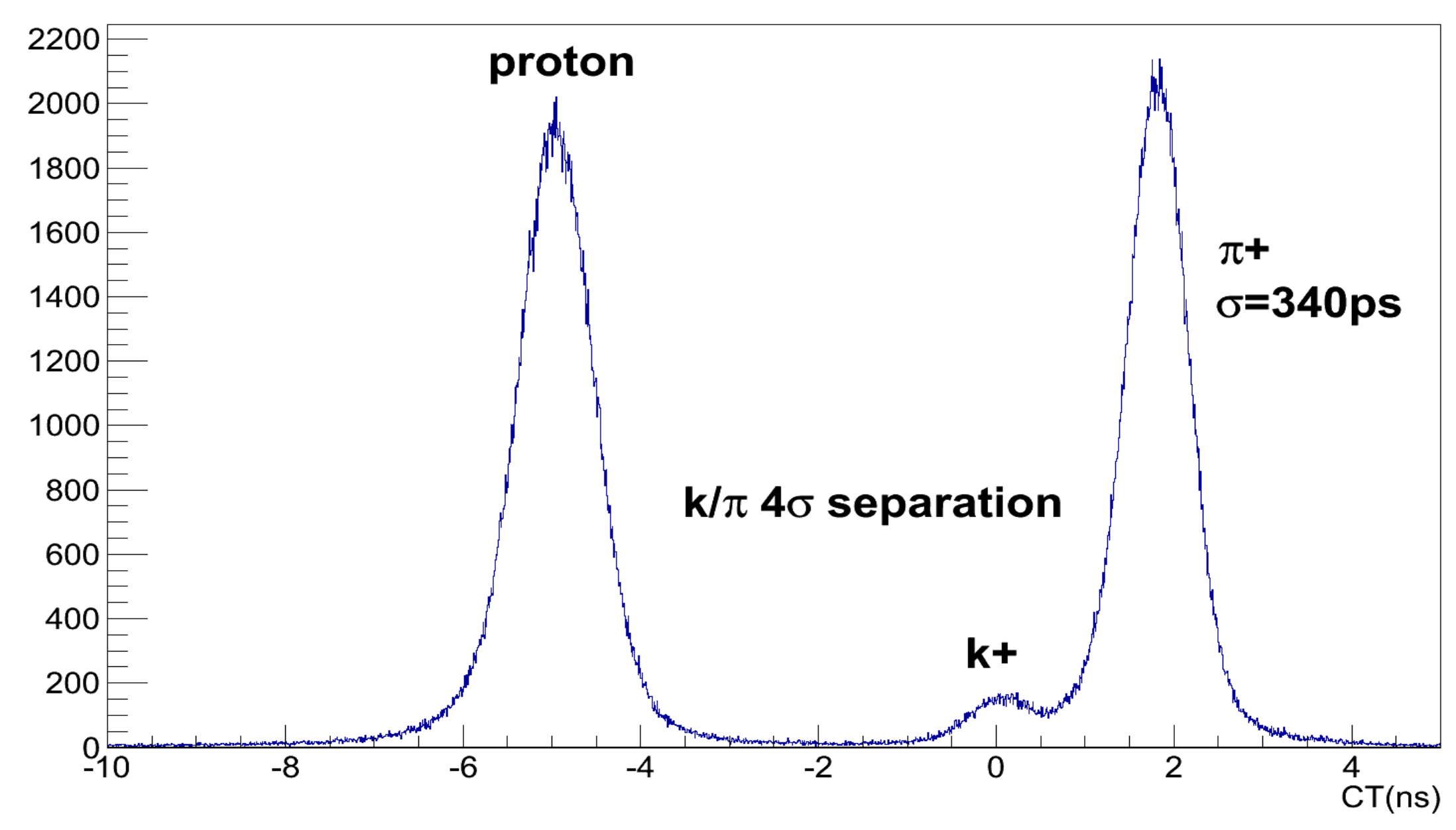

$\mathrm{K}+/ \pi+$ ratio: $\sim 5 \% \quad \mathrm{~K}-/ \pi-$ ratio: $\sim 1 \%$ 


\section{Preliminary $\mathrm{K}+/ \mathrm{K}$ - Collins and Sivers Asymmetries on ${ }^{3} \mathrm{He}$}
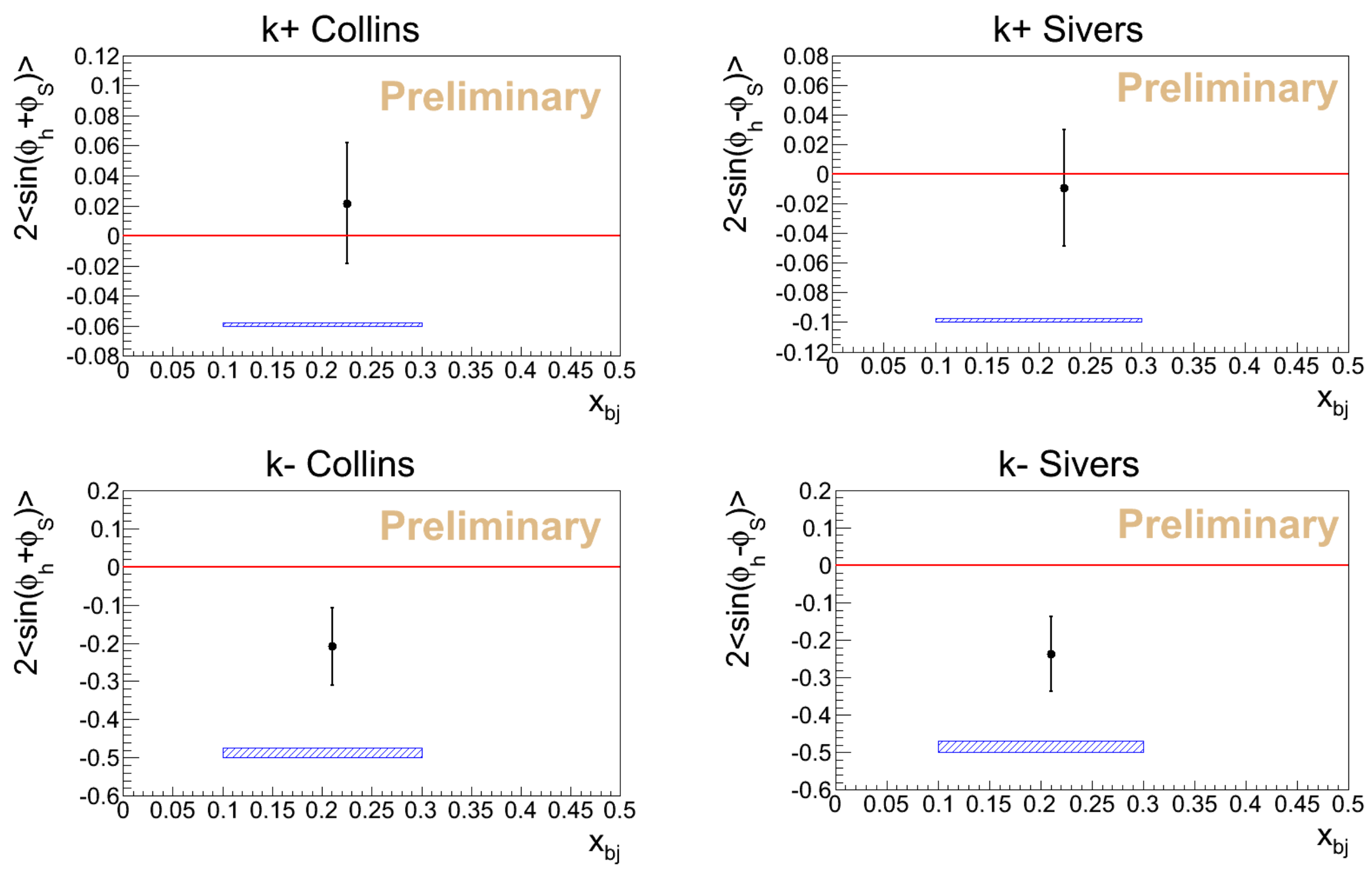


\section{Preliminary New Results (II) from JLab Hall A E06-010 with a Transversely Polarized ${ }^{3} \mathrm{He}(\mathrm{n})$}

\section{Pretzelosity on $\pi+/ \pi-$}

Analysis by Y. Zhang (Lanzhou) and X. Qian (Caltech) 


\section{Extracted Results on Neutron}

Extracted Pretzelosity Asymmetries, $A_{U T} \sin \left(\varphi_{h}-\varphi_{s}\right)$, on the neutron For both $\pi+$ and $\pi-$, consistent with zero within uncertainties.

\section{Preliminary Results}

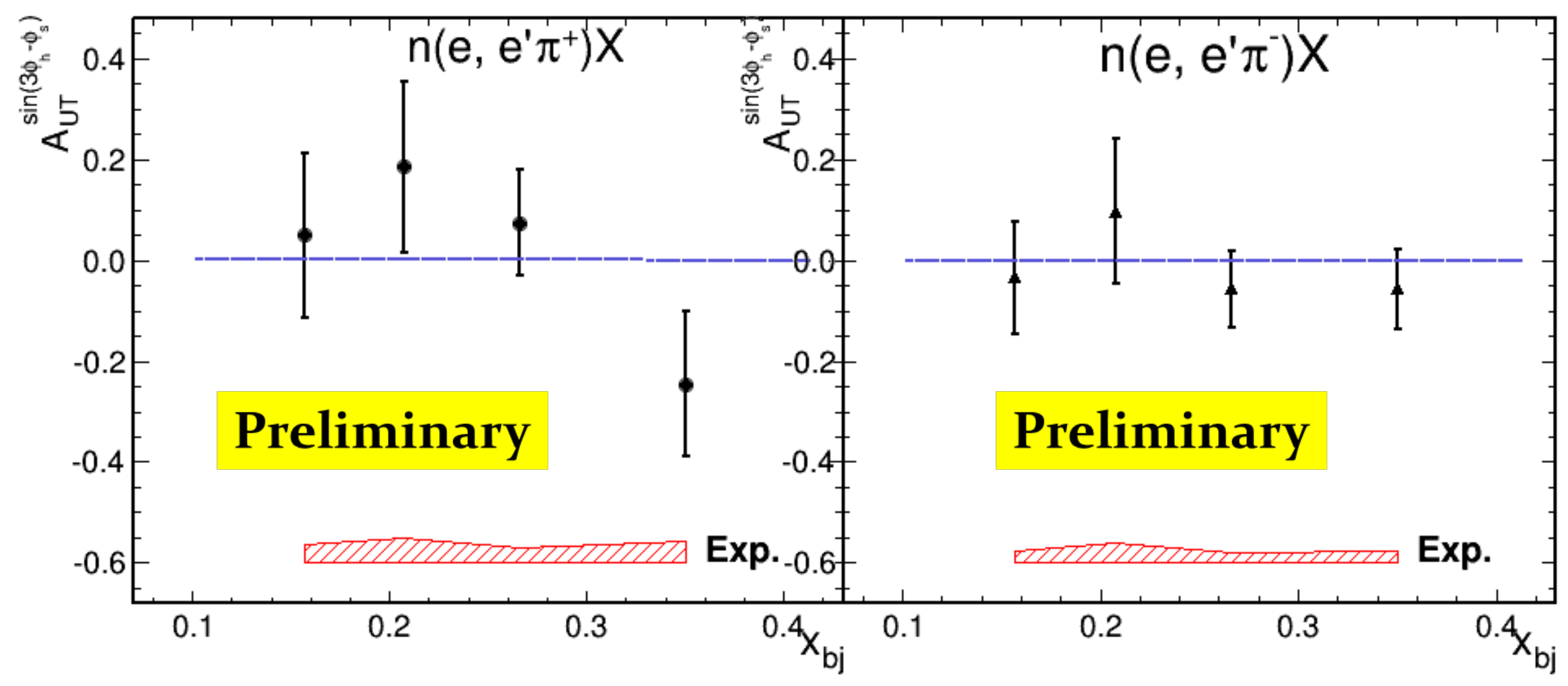




\section{Preliminary New Results (III)from JLab Hall A E06-010 with a polarized ${ }^{3} \mathrm{He}(\mathrm{n})$}

\section{Inclusive Electron SSA}

Analysis by J. Katech(W\&M), X. Qian (Caltech) 


\section{Inclusive Target Single Spin Asymmetry: DIS}

$$
A_{y}\left(Q^{2}\right)=\frac{\sigma^{\uparrow}-\sigma^{\downarrow}}{\sigma^{\uparrow}+\sigma^{\downarrow}}
$$
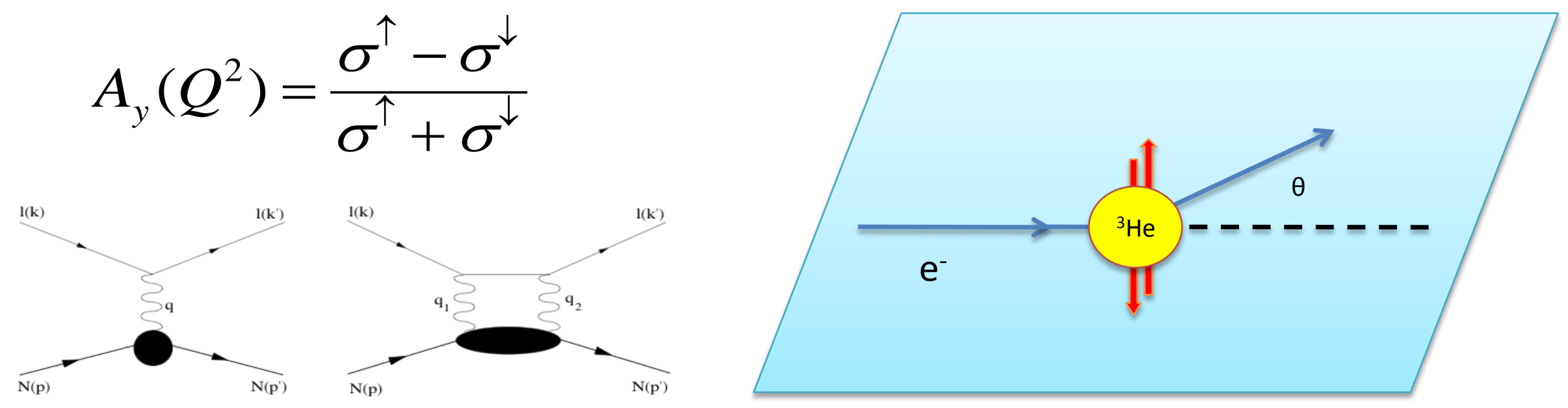

- Unpolarized $\mathrm{e}^{-}$beam incident on ${ }^{3} \mathrm{He}$ target polarized normal to the electron scattering plane.

- However, $A_{y}=0$ at Born level,

$\rightarrow$ sensitive to physics at order $\alpha^{2}$; two-photon exchange.

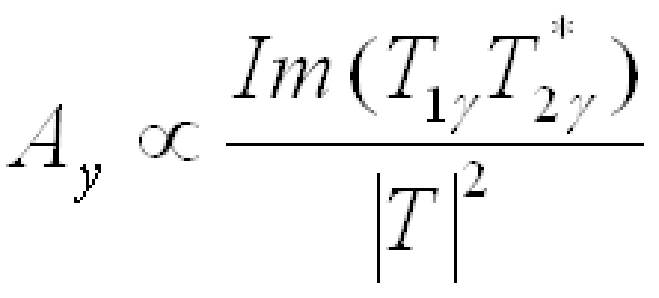

- In DIS case: related to integral of Sivers

- Physics Importance discussed in A. Metz and M. Schlegel's talks (Tuesday) 


\section{Inclusive Target Single-Spin Asymmetry}

\section{SSA in ${ }^{3} \mathrm{He}\left(\mathbf{e}, \mathbf{e}^{\prime}\right)$}

Vertically polarized target

\section{Extracted neutron SSA}

Vertically polarized target
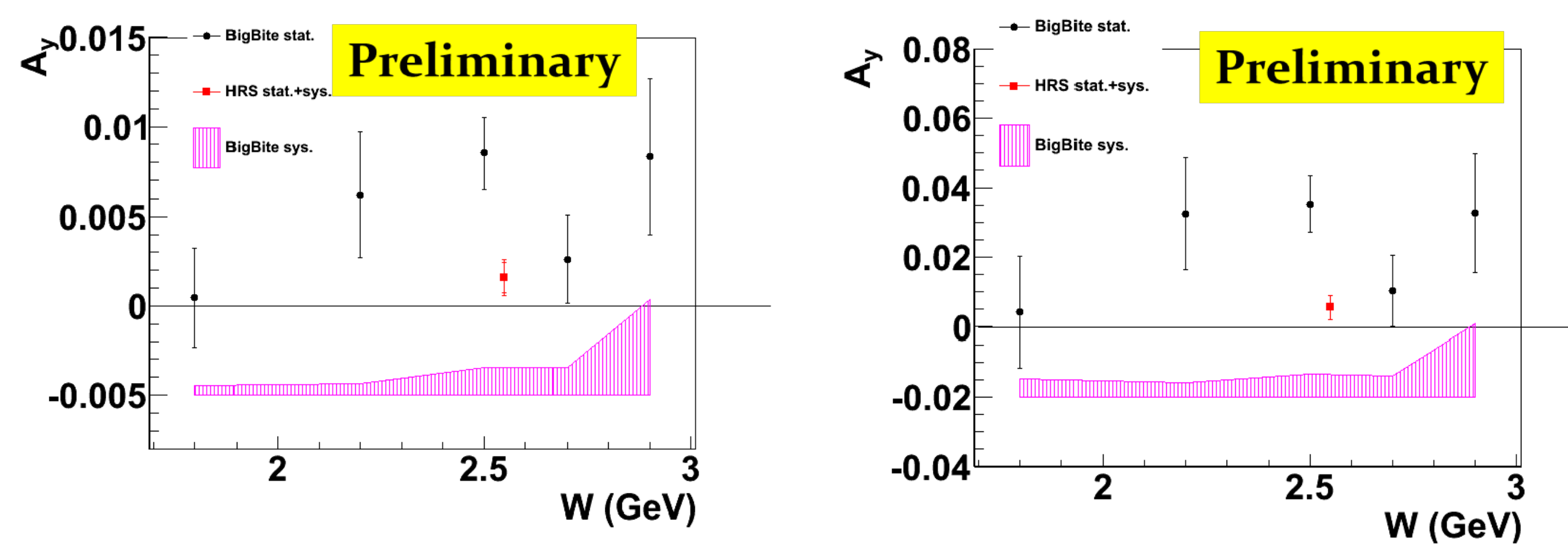


\title{
Preliminary New Results from JLab Hall A E05-015 with a polarized ${ }^{3} \mathrm{He}(\mathrm{n})$
}

\section{Inclusive Electron SSA in Quasi-Elastic Scattering}

\author{
Analysis by Y. Zhang (Rutgers), B. Zhao (W\&M)
}




\section{Incluisve Target Single Spin Asymmetry: QE}

$$
A_{y}\left(Q^{2}\right)=\frac{\sigma^{\uparrow}-\sigma^{\downarrow}}{\sigma^{\uparrow}+\sigma^{\downarrow}}
$$
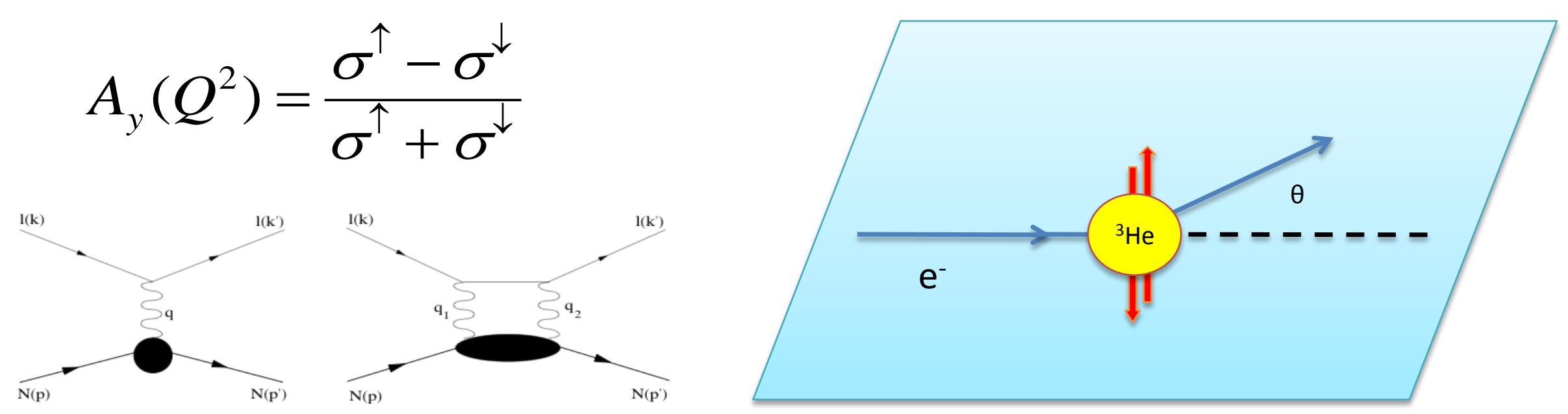

- Unpolarized $\mathrm{e}^{-}$beam incident on ${ }^{3} \mathrm{He}$ target polarized normal to the electron scattering plane.

- However, $A_{y}=0$ at Born level,

$\rightarrow$ sensitive to physics at order $\alpha^{2}$; two-photon exchange.

$A_{y} \propto \frac{\operatorname{Im}\left(T_{1 \gamma} T_{2 \gamma}^{*}\right)}{|T|^{2}}$

- (Q)Elastic: Calculable at large $Q^{2}$ using moments of GPD's

- Measurement of $A_{y}$ at large $Q^{2}$ provides access to GPD's 


\section{Preliminary ${ }^{3} \mathrm{He}$ results at $\mathrm{Q}^{2}=0.5$ and $1.0 \mathrm{GeV}^{2}$}

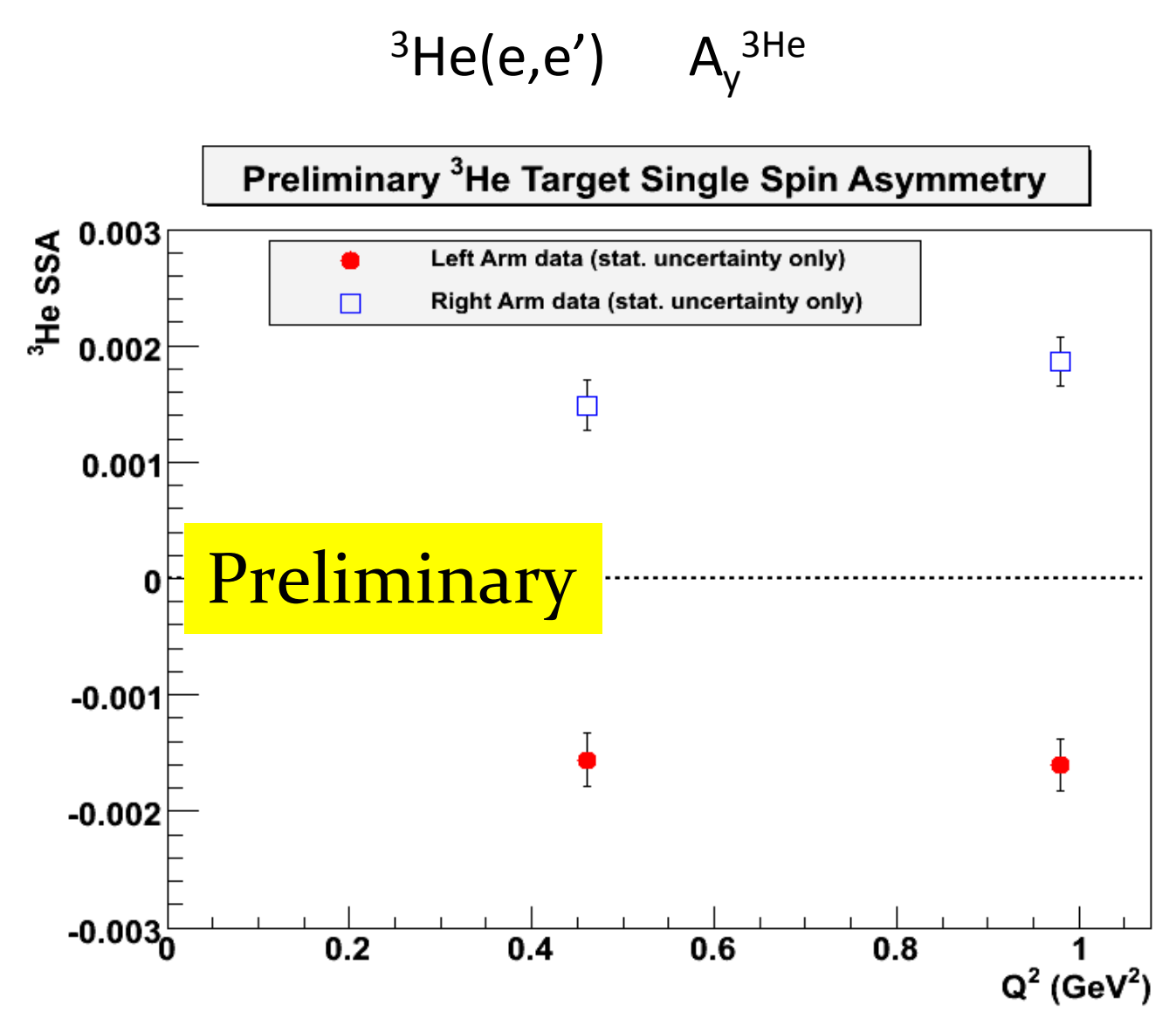

Data above is for helium-3

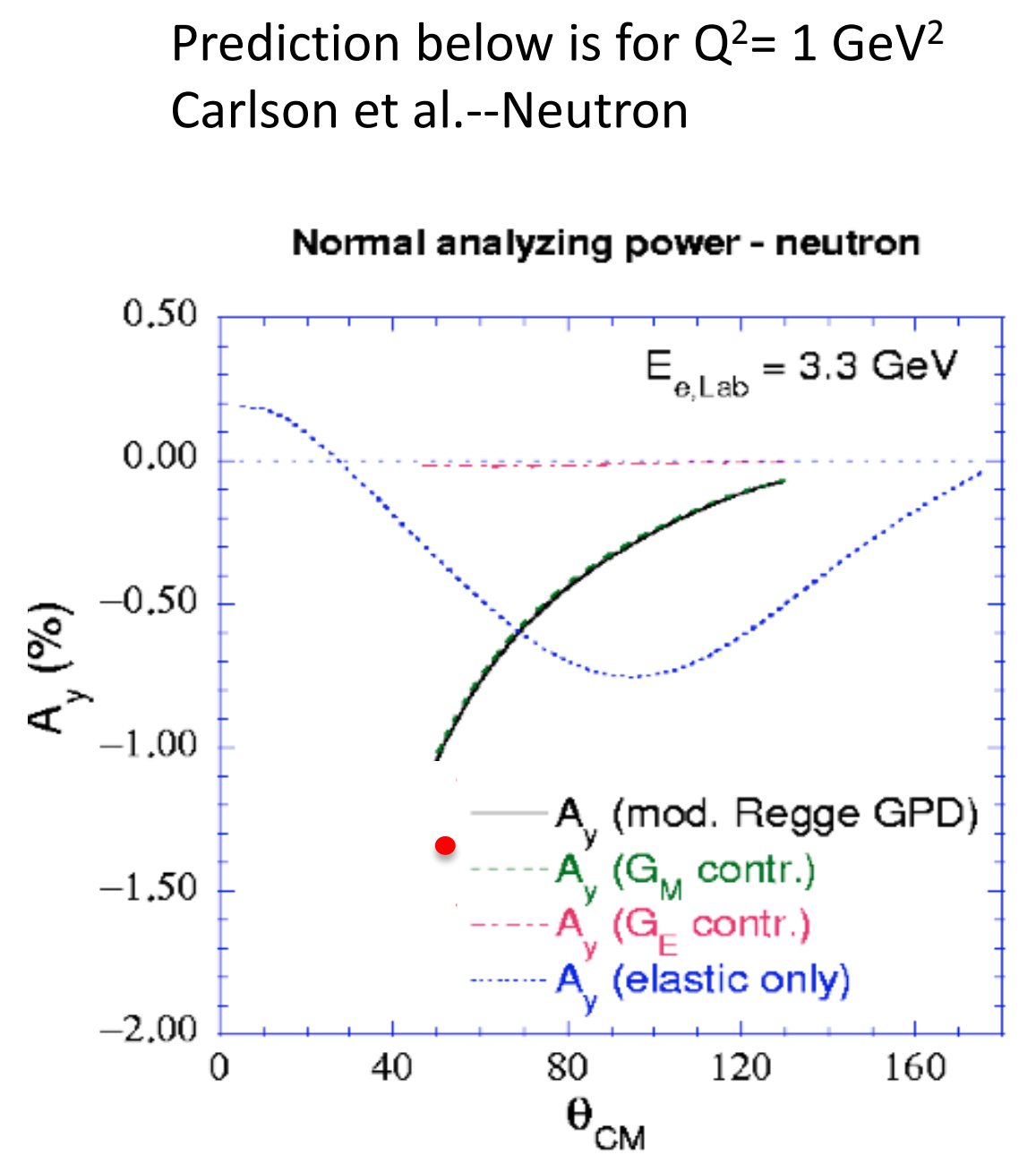




\section{Preliminary New Results (IV) from JLab Hall A E06-010 with a transversely polarized ${ }^{3} \mathrm{He}(\mathrm{n})$}

\section{Inclusive Hadron SSA}

Analysis by K, Allada (JLab), Y. Zhao (USTC) 


\section{Inclusive Hadron Electroproduction}

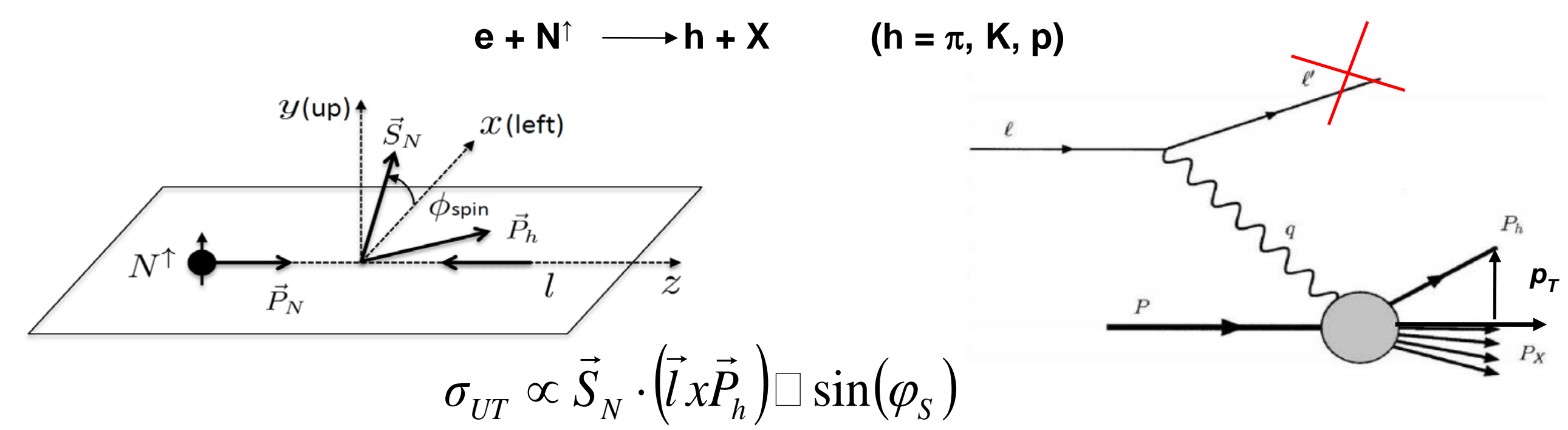

Why a non-zero $A_{N}$ is interesting?

- Analogues to $A_{N}$ in $p p^{\uparrow} \rightarrow h X$ collision

$$
A_{N}\left(x_{F}, p_{T}\right)=A_{U T}^{\sin \left(\varphi_{S}\right)}
$$

- Simpler than $p p^{\uparrow} \rightarrow h X$ due to only one quark channel

- Same transverse spin effects as SIDIS and $p-p$ collisions (Sivers, Collins, twist-3)

- Clean test TMD formalism (at large $p_{T} \sim 1 \mathrm{GeV}$ or more)

- To help understand mechanism behind large $A_{N}$ in $p p^{\uparrow} \rightarrow h X$ in the TMD framework 


\section{Transverse SSA in Inclusive Hadron}

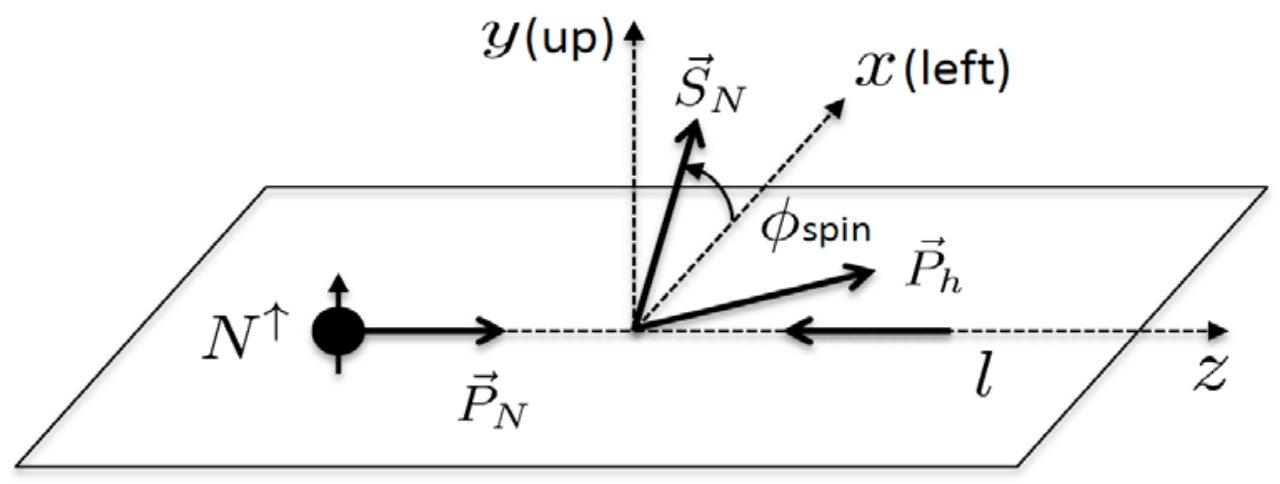

$\vec{S}_{N} \cdot\left(\vec{l} \times \vec{P}_{h}\right)=0$

$$
A_{U T}^{\sin \left(\varphi_{S}\right)}=\frac{N^{\uparrow}-N^{\downarrow}}{N^{\uparrow}+N^{\downarrow}}
$$

- Target spin flip every 20 minutes

- Acceptance effects cancels

- Overall systematic check with $A_{N}$ at $\phi_{S}=0$

- False asymmetry $<0.1 \%$

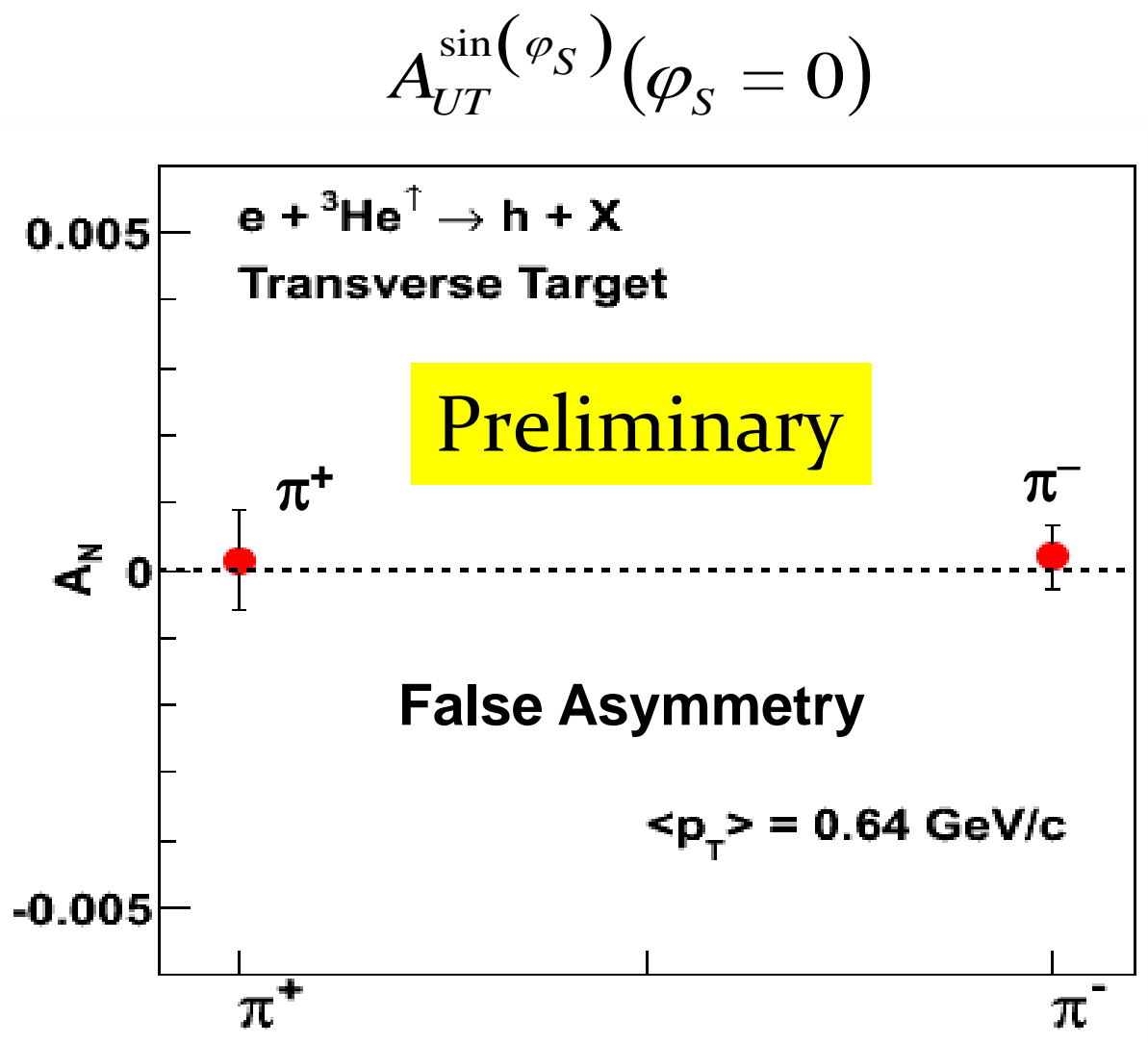




\section{E06-010: Inclusive Hadron SSA $\left(A_{N}\right)$}

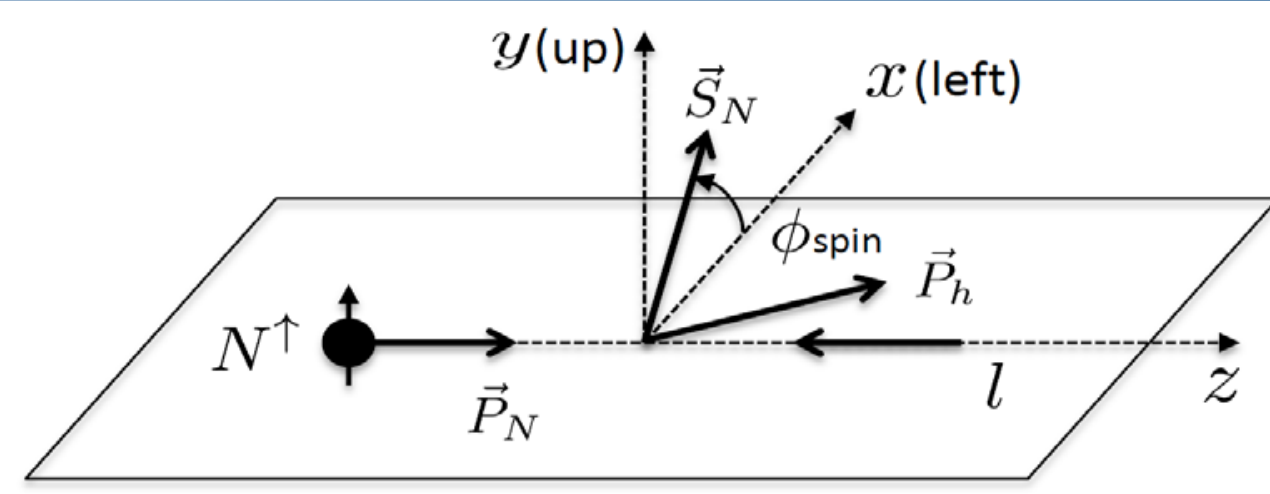

$$
\vec{S}_{N} \cdot\left(\vec{I} \times \vec{P}_{h}\right) \neq 0
$$

- Clear non-zero target SSA

- Opposite sign for $\pi^{+}$and $\pi^{-}$

$$
A_{U T}^{\sin \left(\varphi_{S}\right)}\left(\varphi_{S}=90^{\circ}\right)
$$

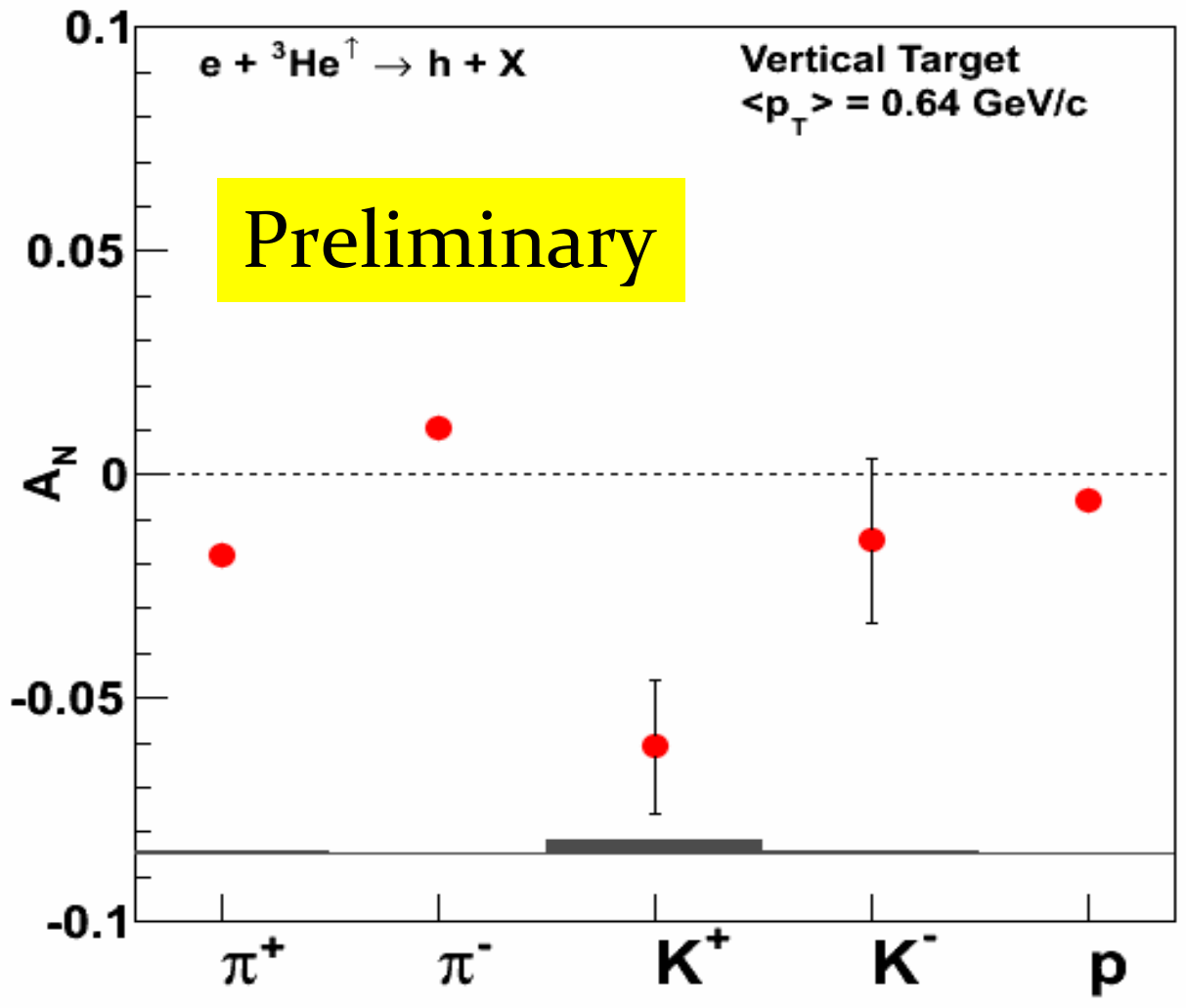




\section{E06-010: Inclusive Hadron SSA $\left(A_{N}\right)$}

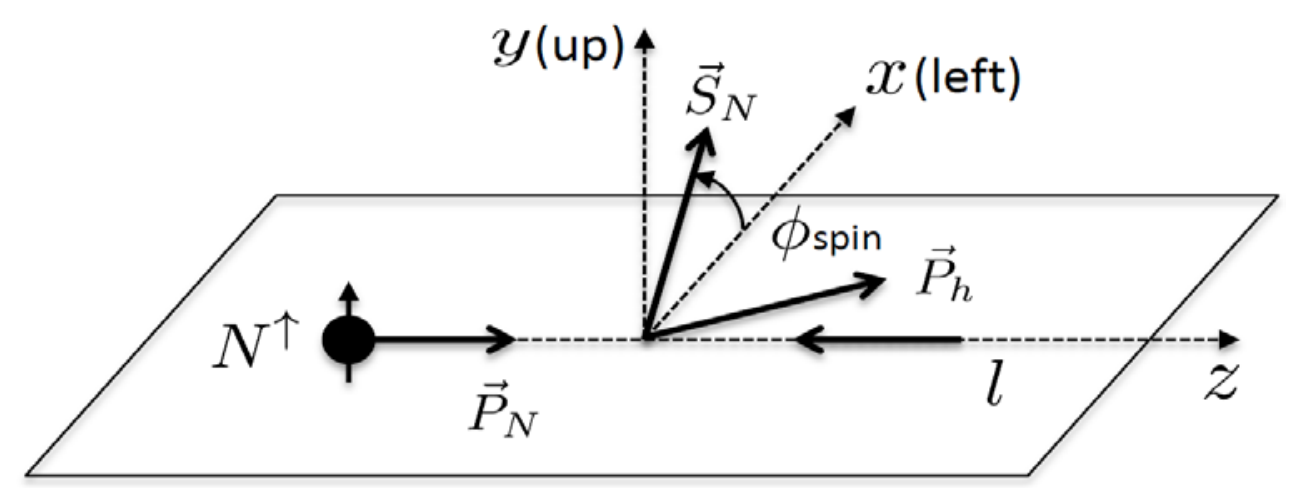

$$
\vec{S}_{N} \cdot\left(\vec{I} x \vec{P}_{h}\right) \neq 0
$$

- Clear non-zero target SSA

- Opposite sign for $\pi^{+}$and $\pi^{-}$

- $A_{N}$ at low $p_{T}$ not very well understood
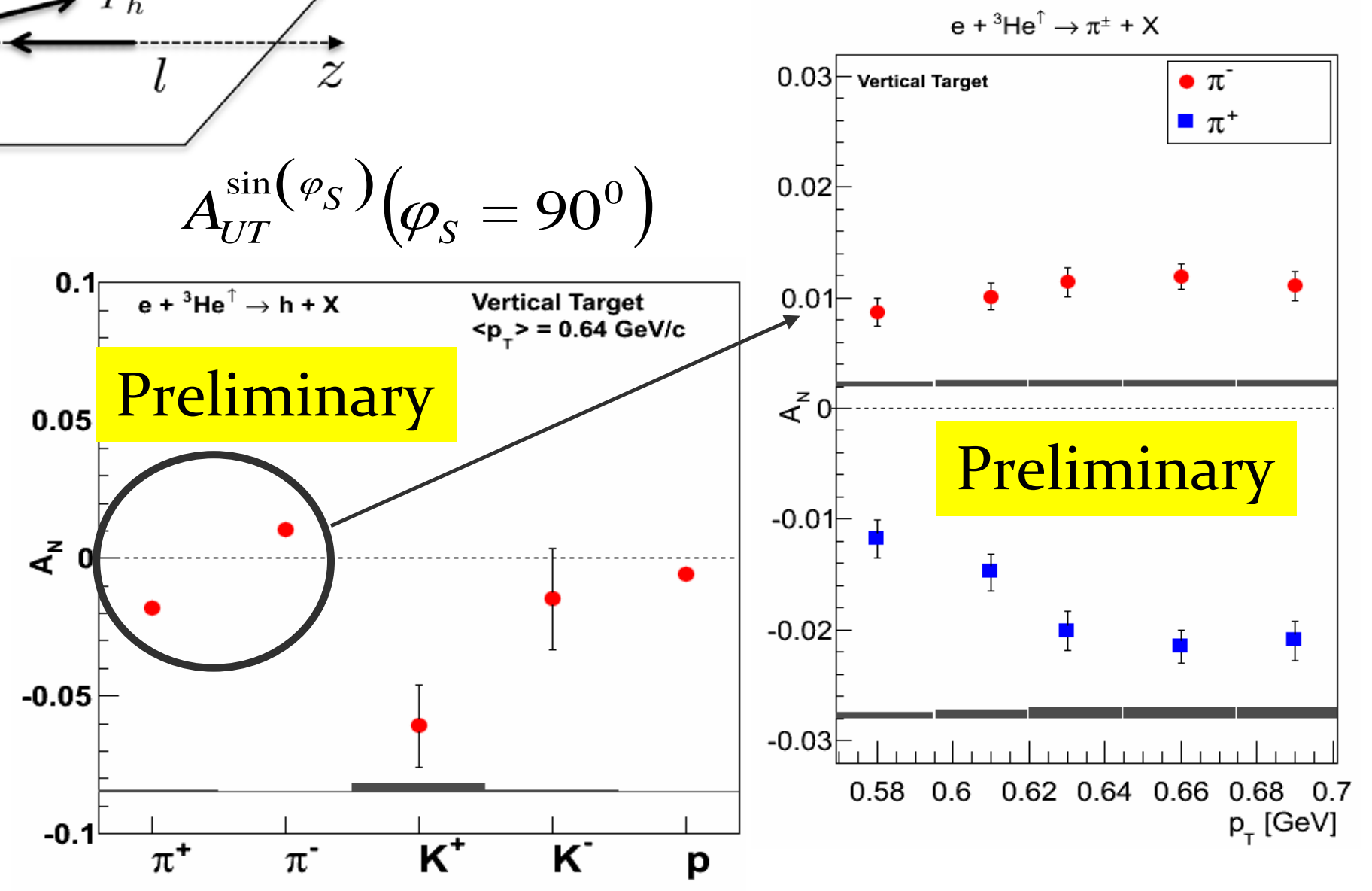


\title{
Future: TMD study with SoLID at $12 \mathrm{GeV}$ JLab Hall A
}

Precision 4-D mapping of Collins/Sivers/Pretzelosity

\author{
Worm-Gear I/II
}

with Polarized ${ }^{3} \mathrm{He}$ (Neutron) and Proton 


\section{JLab 12 GeV Era: Precision Study of TMDs}

- From exploration to precision study with $12 \mathrm{GeV}$ JLab

- Transversity: fundamental PDFs, tensor charge

- TMDs: 3-d momentum structure of the nucleon

- $\rightarrow$ Quark orbital angular momentum

- Multi-dimensional mapping of TMDS

- 4-d $\left(x, z, P_{\perp}, \mathrm{Q}^{2}\right)$

- Multi-facilities, global effort

- Precision $\rightarrow$ high statistics

- high luminosity and large acceptance 


\section{SoLID for SIDIS/PVDIS with $12 \mathrm{GeV}$ JLab}

- Exciting physics program:

Five approved experiments:

three SIDIS "A rated", one PVDIS "A rated", one J/Psi "A- rated"

- International collaboration: eight countries and 50+ institutions

- CLEOII Magnet

- GEMs for tracking

- Cherenkov and EM

Calorimeter for electron PID

- Heavy Gas Cherenkov and MRPC (TOF) for pion PID

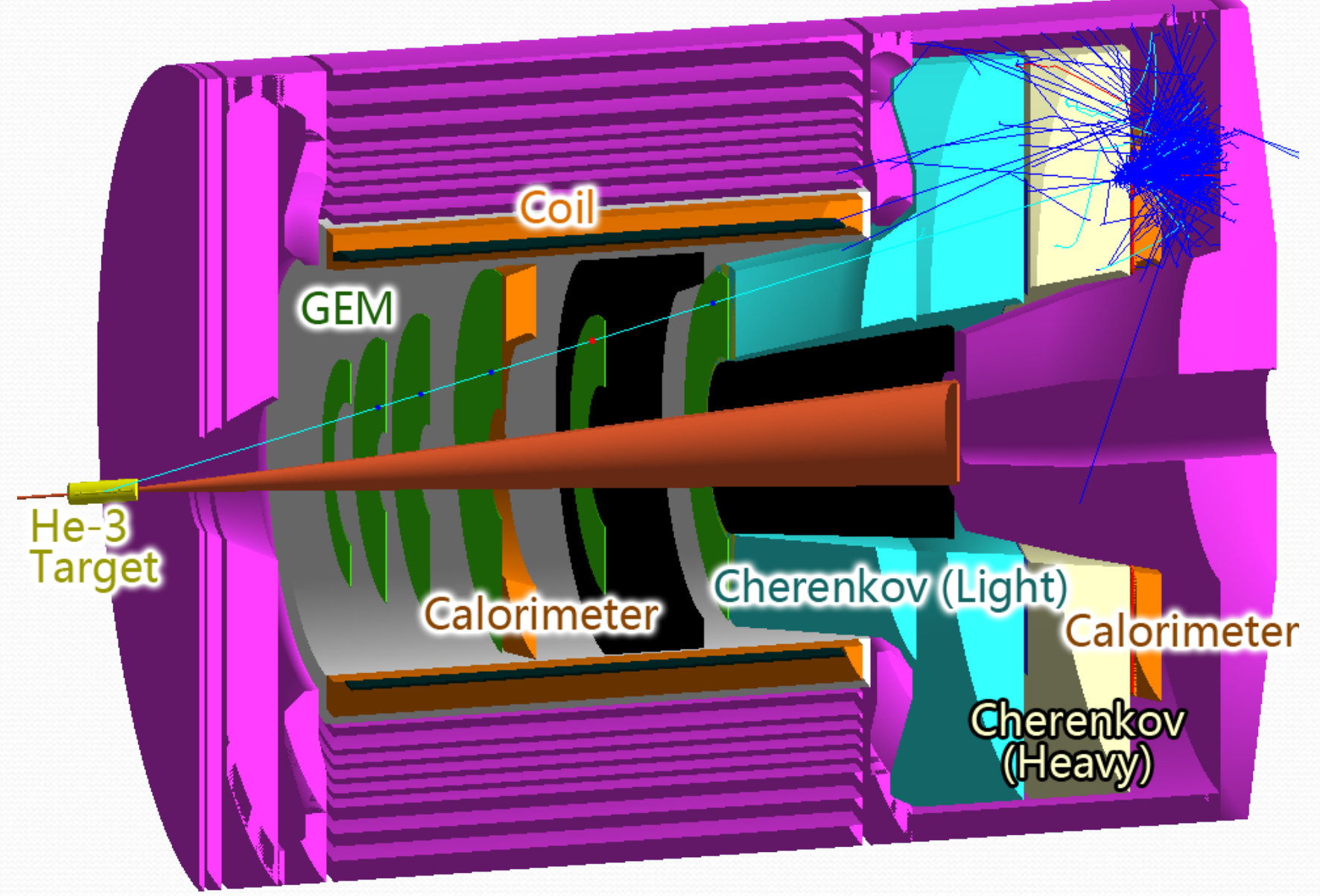




\section{E12-10-006/E12-11-108, Both Approved with "A" Rating Mapping of Collins(Sivers) Asymmetries with SoLID}

- Both $\pi+$ and $\pi-$

Collins Asymmetry

- Precision Map in region

$x(0.05-0.65)$

$z(0.3-0.7)$

$\mathrm{Q}^{2}(1-8)$

$\mathrm{P}_{\mathrm{T}}(0-1.6)$

- $<10 \%$ d quark tensor charge

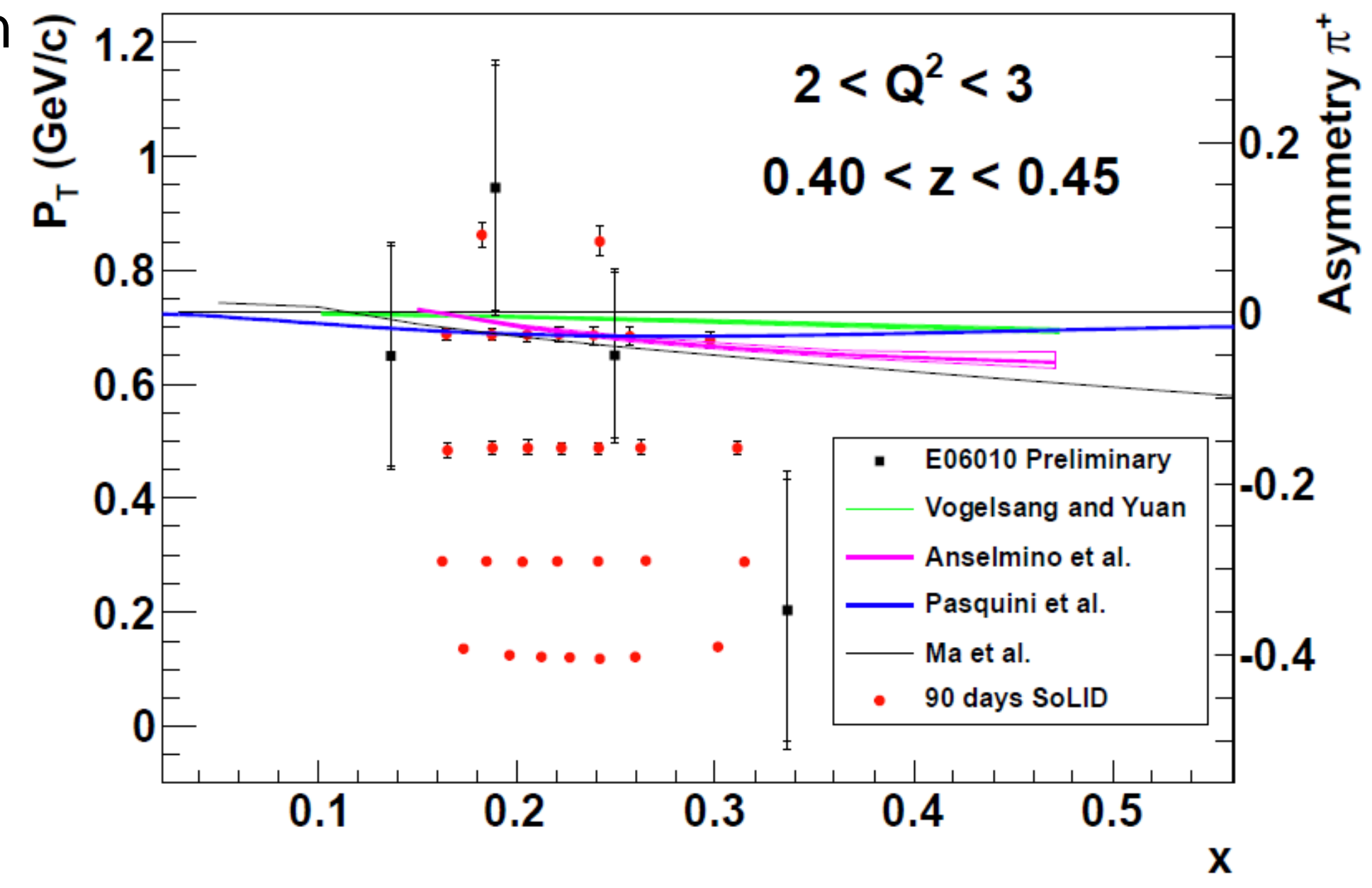




\section{Map Collins and Sivers asymmetries in 4-D $\left(\mathrm{X}, \mathrm{z}, \mathrm{Q}^{2}, \mathrm{P}_{\mathrm{T}}\right)$}

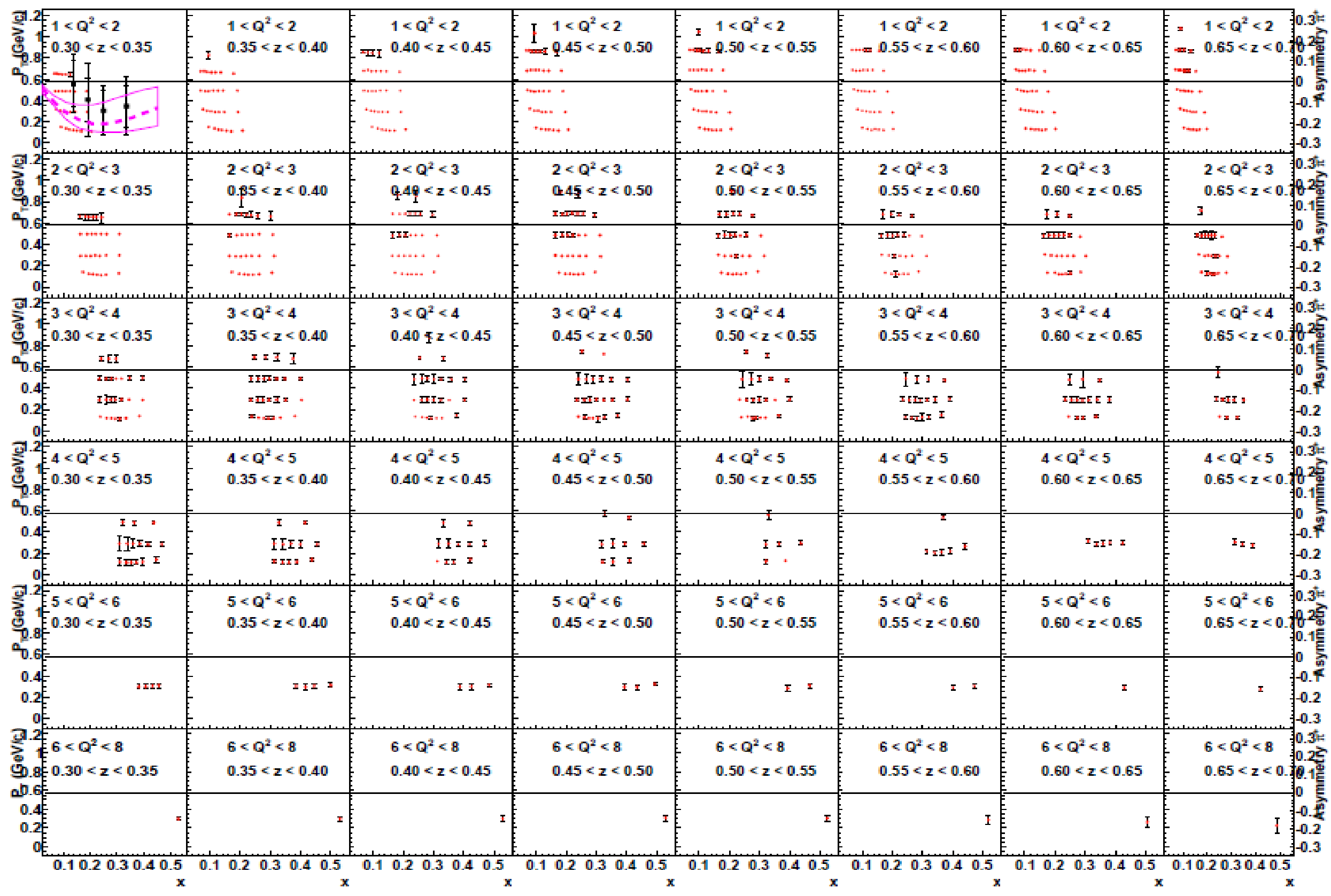




\section{E12-11-107: Worm-gear functions (" $\mathrm{A}$ ' rating: )}

Spokespersons: Chen/Huang/Qiang/Yan

$h_{11}=\Theta-\infty$
$g_{1 T}=\Theta-\Theta$

- Dominated by real part of interference between $L=0(S)$ and $L=1(P)$ states

- No GPD correspondence

- Lattice QCD -> Dipole Shift in mom. space.

- Model Calculations $->h_{1 \mathrm{~L}}{ }^{\perp}=$ ? $-g_{1 \mathrm{~T}}$.

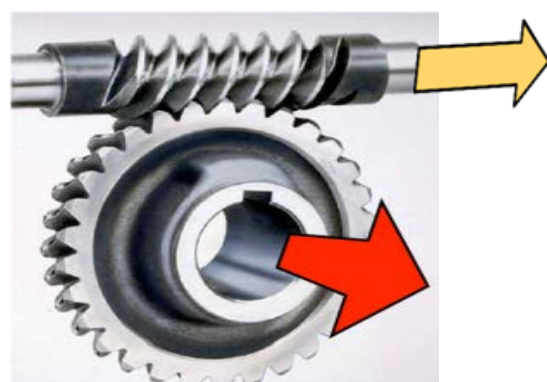

Longi-transversity

Trans-helicity

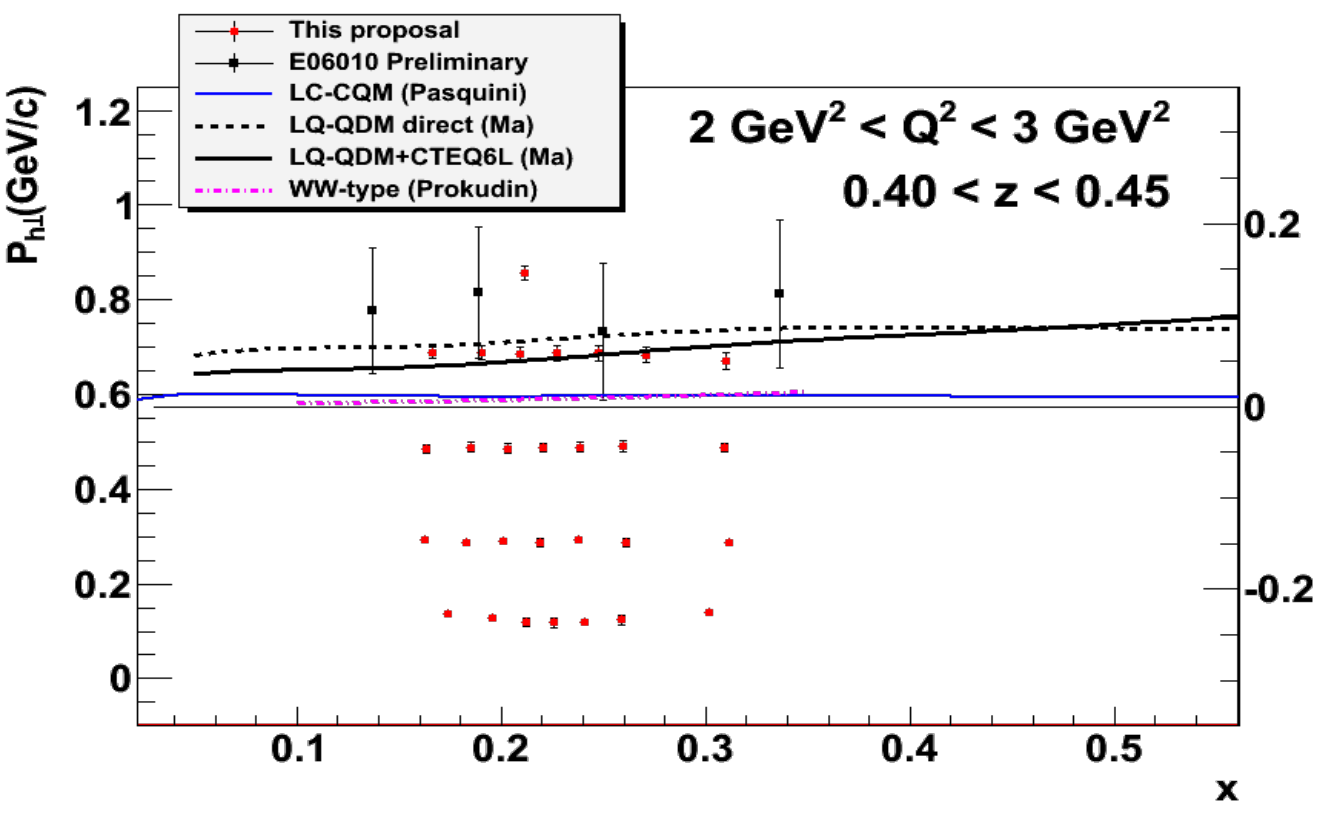

$A_{L T} \sim g_{1 T}(x) D_{1}(z)$

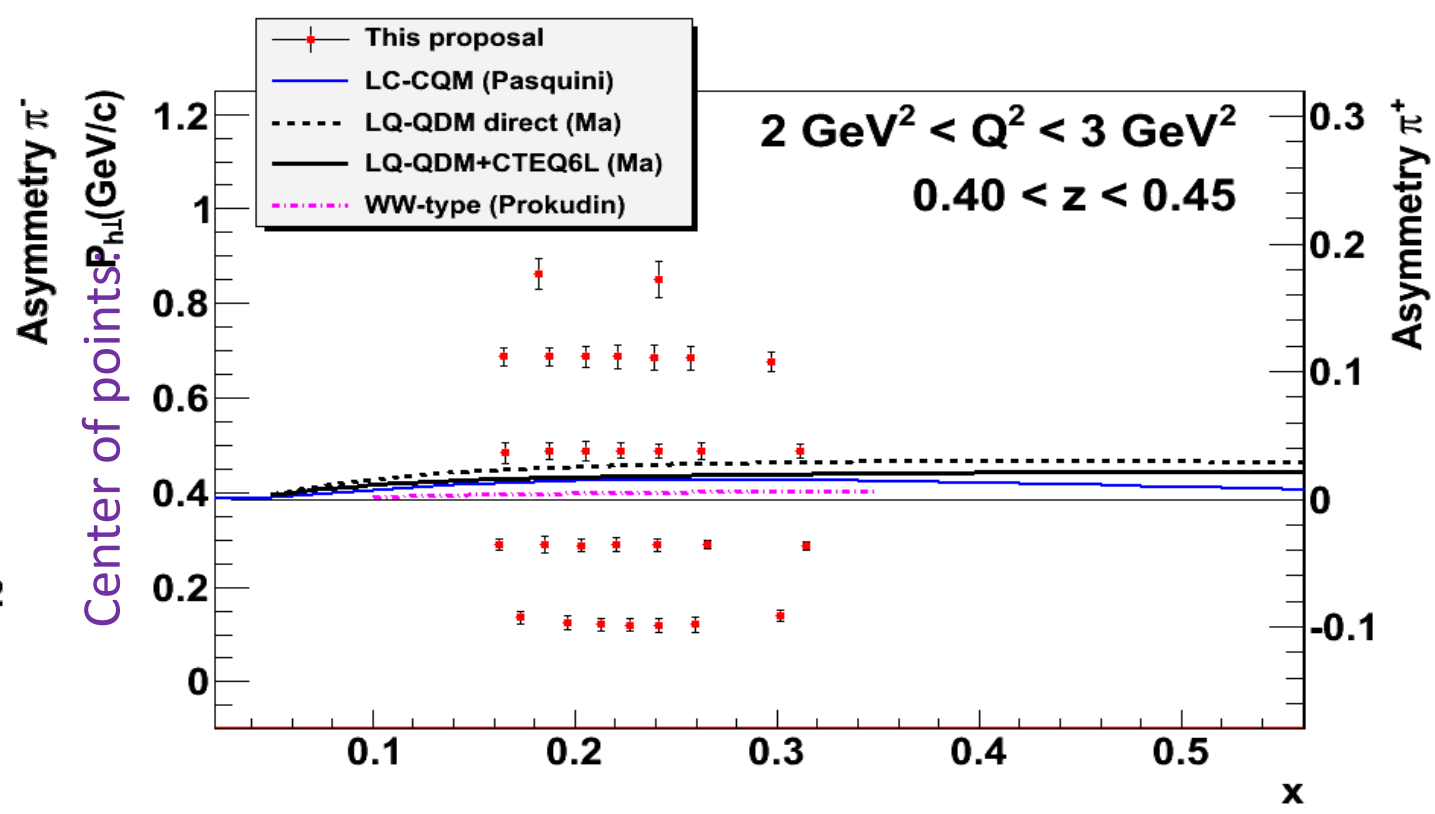

$A_{U L} \sim h^{\perp}{ }_{1 L}(x) H^{\perp}{ }_{1}(z)$ 


\section{Future: TMD study with SoLID at $12 \mathrm{GeV}$ JLab Hall A}

New Letter Of Intent: Dihadron Production 


\section{Measure Transversity via Dihadron with SoLID}

Lol submitted to Jlab PAC 40, J. Zhang, A. Courtoy, et al.

- Precision dihadron $(\pi+l \pi-)$ production on a transversely polarized ${ }^{3} \mathrm{He}(\mathrm{n})$

- Extract transversity on neutron

- Provide crucial inputs for flavor separation of transversity

talk by M.Radici
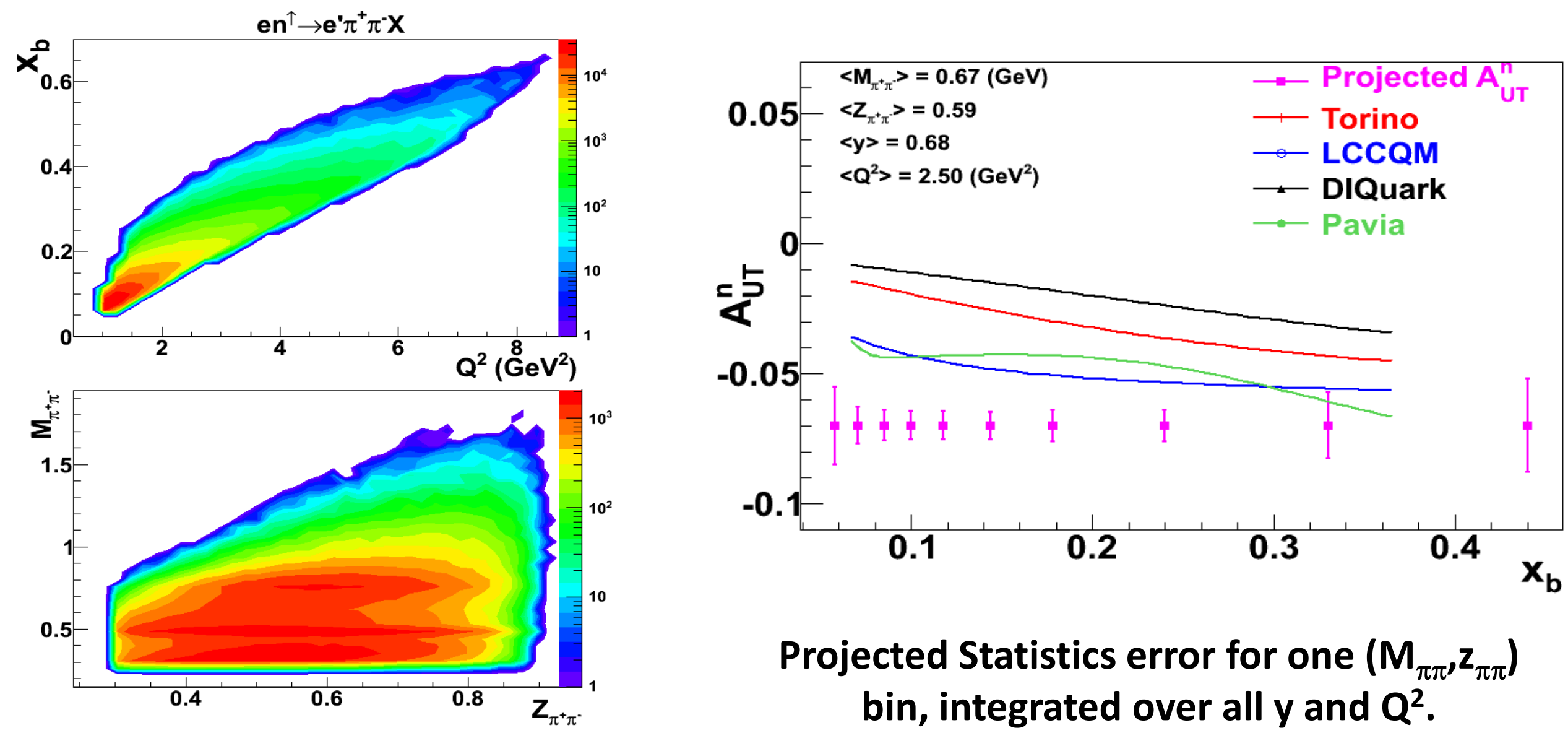

Projected Statistics error for one $\left(\mathrm{M}_{\pi \pi}, \mathbf{z}_{\pi \pi}\right)$ bin, integrated over all $y$ and $Q^{2}$.

Wide $x_{b}$ and $Q^{2}$ coverages 


\section{Projected Statistics Error}

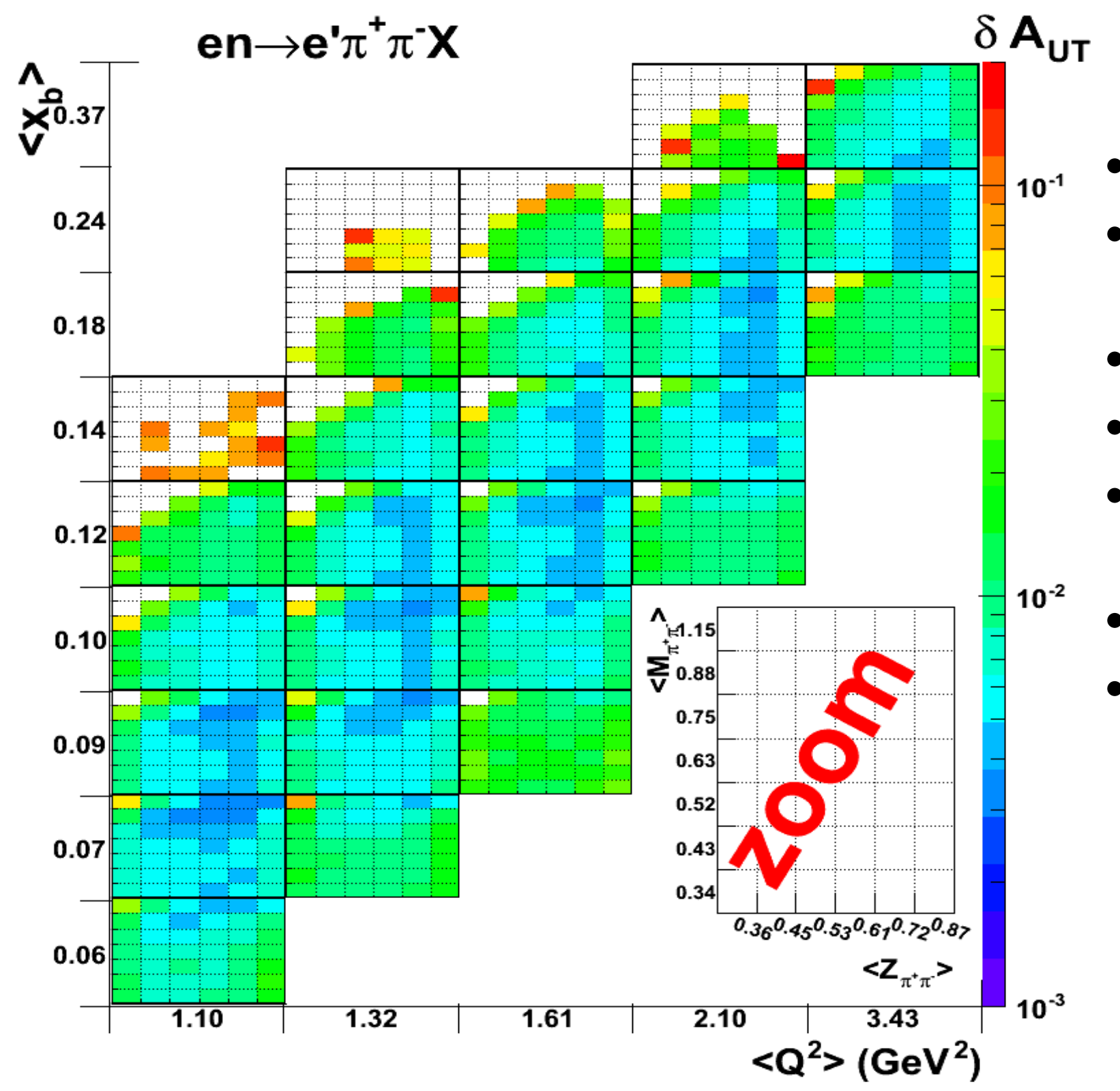

- Hall A, SoLID program

- Polarized ${ }^{3} \mathrm{He}$ target, ( $60 \%$ polarization)

- Lumi $=10^{36}(\mathrm{n}) / \mathrm{s} / \mathrm{cm}^{2}$

- Wide $x_{b}$ and $Q^{2}$ coverages

- Bin central values labeld on axises

- 4-d $\left(x_{b}, Q^{2}, Z_{\pi+\pi-}, M_{\pi+\pi-}\right)$ mapping

- Z scale (color) represent stat. error 


\section{Summary on SoLID TMD Program}

- Unprecedented precision 4-d mapping of SSA

- Collins, Sivers, Pretzelosity and Worm-Gear

- Both polarized ${ }^{3} \mathrm{He}(\mathrm{n})$ and polarized proton with SoLID

- Study factorization with $x$ and $z$-dependences

- Study $P_{T}$ dependence

- Combining with the world data

- extract transversity and fragmentation functions for both $u$ and $d$ quarks

- determine tensor charge

- study TMDs for both valence and sea quarks

- learn quark orbital motion and quark orbital angular momentum

- study $Q^{2}$ evolution

- Global efforts (experimentalists and theorists), global analysis

- much better understanding of multi-d nucleon structure and QCD

- Long-term future: EIC to map sea and gluon SSAs 


\section{Long-term Future: TMD study with EIC}

MEIC@JLab and E-RHIC@BNL

New Opportunity: EIC in China 


\section{Image the Transverse Momentum of the Quarks}

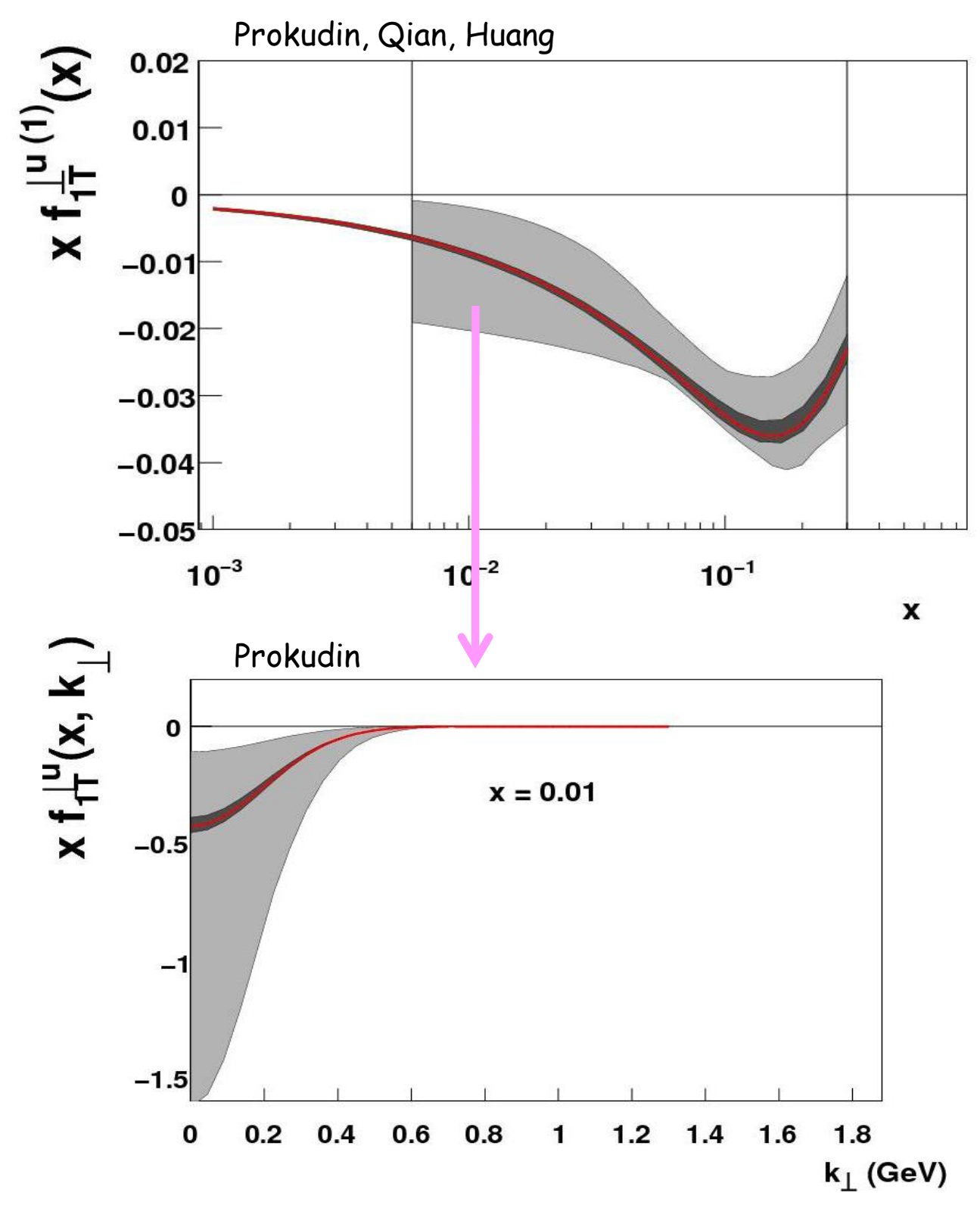

Only a small subset of the $\left(x, Q^{2}\right)$ landscape has been mapped here.

An EIC with good luminosity \& high transverse polarization is the optimal tool to to study this!

Exact $k_{T}$ distribution presently essentially unknown! 


\section{Lepton-Nucleon Facilities}

EIC@HIAF: $e(3 \mathrm{GeV})+p(12 \mathrm{GeV})$, both polarized, $L(\max )=10^{33} \mathrm{~cm}^{2} / \mathrm{s}$

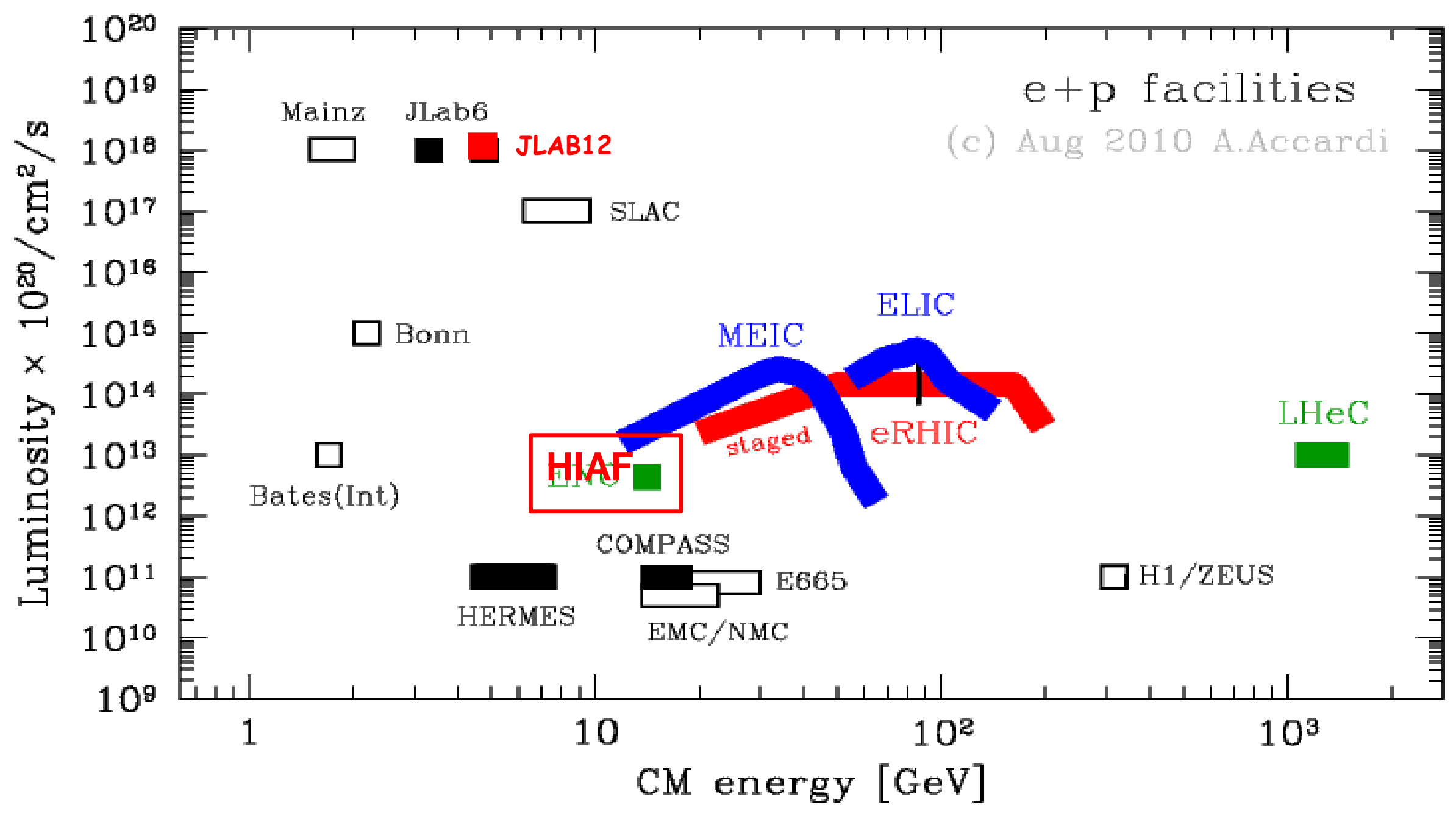




\section{High Intensity Accelerator Facility (One Option)}

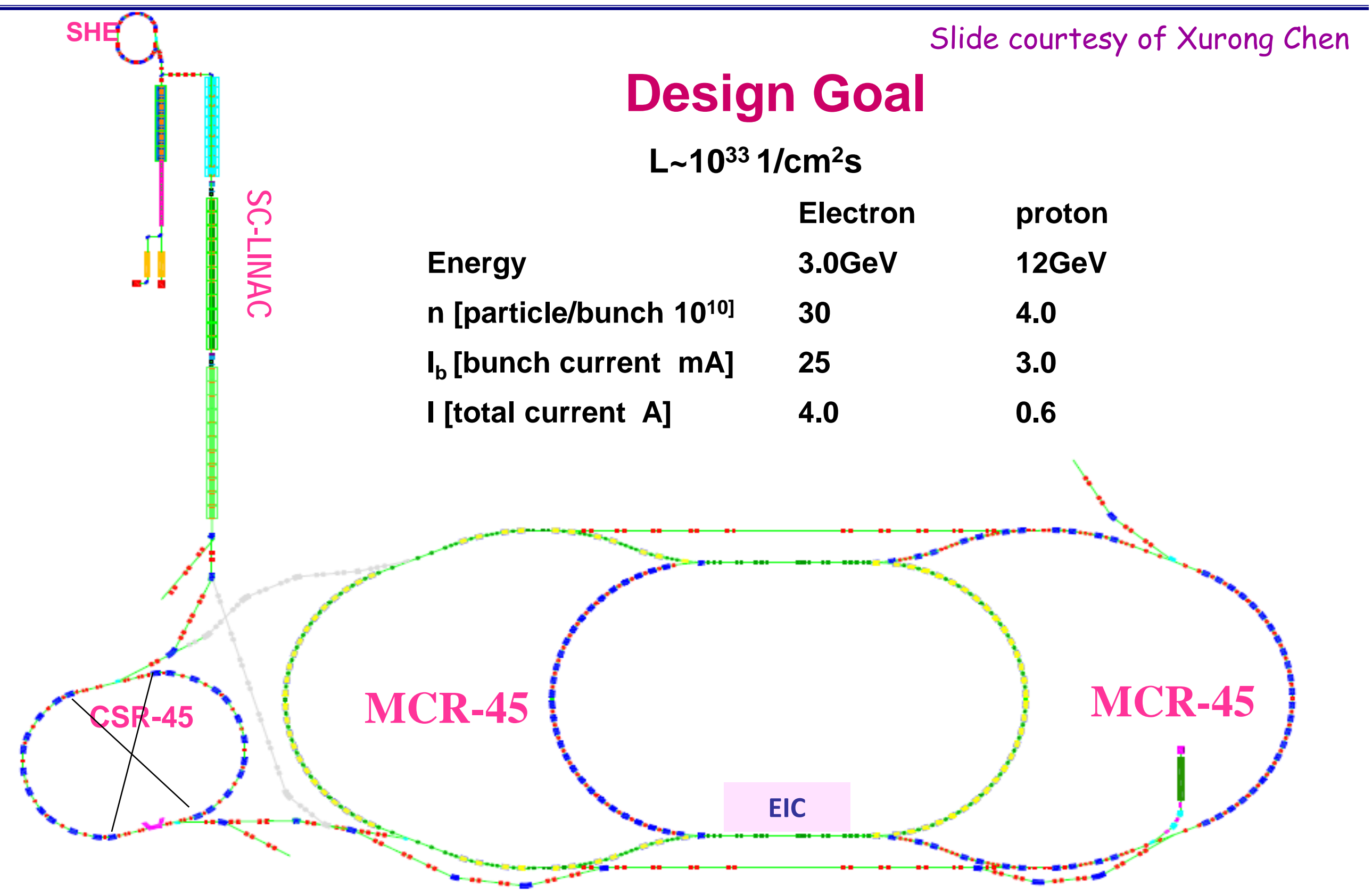




\section{EIC@HIAF Kinematic Coverage Comparison with JLab 12 GeV}

$$
\mathrm{e}(3 \mathrm{GeV})+\mathrm{p}(12 \mathrm{GeV}) \text {, both polarized, } \mathrm{L}(\max )=10^{33} \mathrm{~cm}^{2} / \mathrm{s}
$$

\section{EIC@HIAF:}

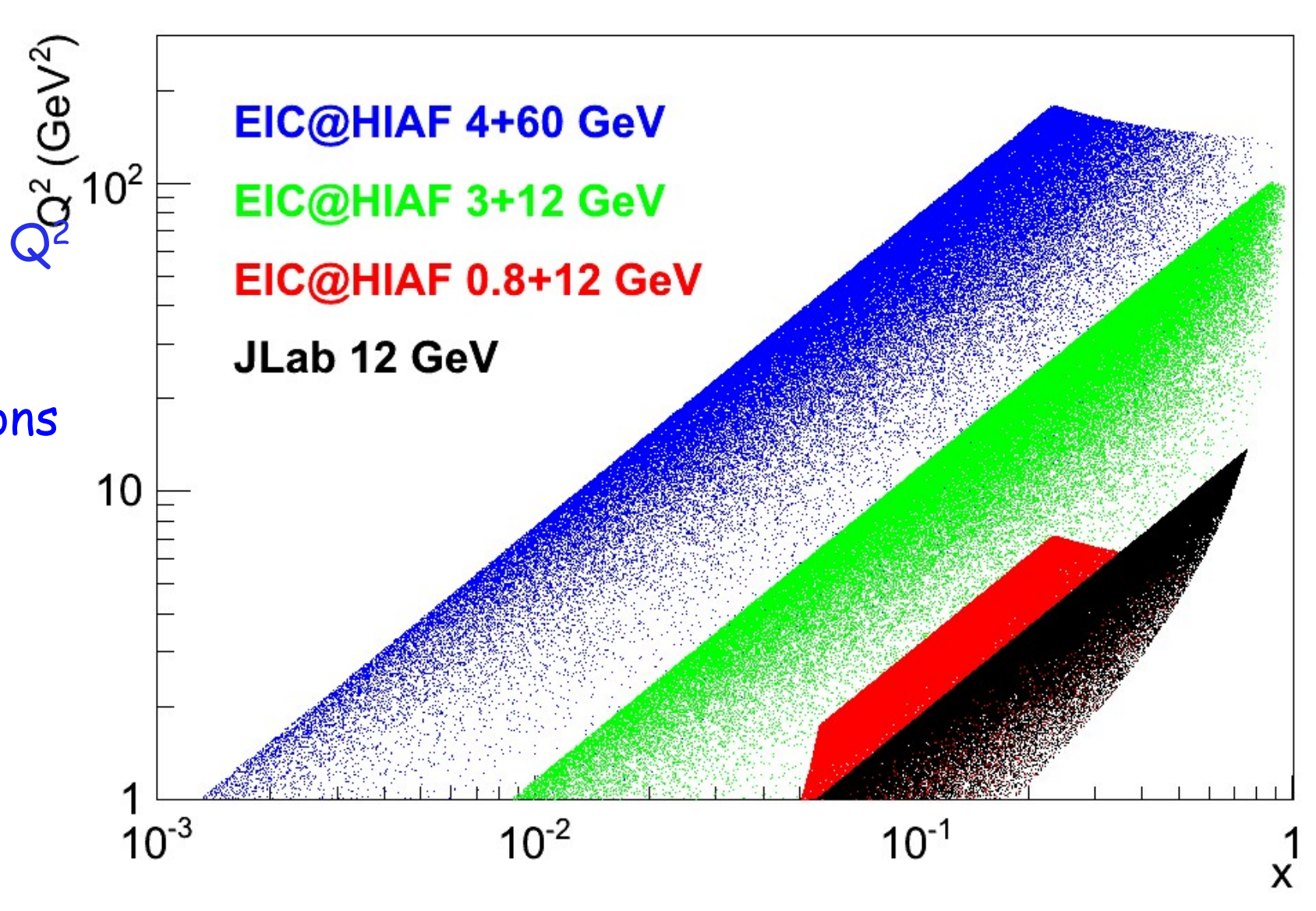

- Timeline: Funding Approved for HIAF EIC under design/discussion Construction 2014-2019

- study sea quarks $(x>0.01)$

- deep exclusive scattering at $Q^{2}$ $>$ 5-10

- higher $Q^{2}$ in valance region

- range in $Q^{2}$ allows study gluons 


\section{Science Goals}

\section{The Science of eRHIC/MEIC}

Goal: Explore and Understand QCD:

Map the spin and spatial structure of quarks and gluons in nucleons

Discover the collective effects of gluons in atomic nuclei

(role of gluons in nuclei \& onset of saturation)

Emerging Themes:

Understand the emergence of hadronic matter from quarks and gluons \& EW

\section{The Science of EIC@HIAF}

One Main Goal: Explore Hadron Structure

Map the spin-flavor, multi-d spatial/momentum structure of valence \& sea quarks 


\section{TMD Study and other Programs at EIC@HIAF}

- Unique opportunity for TMD in "sea quark" region reach $x \sim 0.01$ (JLab12 mainly valence quark region, reach $x \sim 0.1$ )

- Significant increase in $\mathrm{Q}^{2}$ range for valence region energy reach $\mathrm{Q}^{2} \sim 40 \mathrm{GeV}^{2}$ at $\mathrm{x} \sim 0.4 \quad\left(\mathrm{JLab12}, \mathrm{Q}^{2}<10\right)$

- Significant increase in $P_{T}$ range reach $>1 \mathrm{GeV}$ ? (TMD/co-linear overlap region) (JLab12, reach $<1 \mathrm{GeV}$ )

- Other Physics Programs:

Nucleon spin-flavor structure (polarized sea, $\Delta s$ )

3-d Structure: GPDs (DVMP, pion/Kaon)

e-A to study hadronization

Pion/Kaon structure functions?

$2^{\text {nd }}$ Conference on QCD and Hadron Physics: http://qcd2013.csp.escience.cn/dct/page/1 Whitepaper on EIC@China is being worked on: need inputs and help from international community 


\section{Summary}

- SSA and TMD study have been exciting and fruitful

- Recent and Preliminary Results from JLab Hall A with a transversely polarized ${ }^{3} \mathrm{He}(\mathrm{n})$ target

Collins/Sivers asymmetries for $\pi+/ \pi-/ \mathrm{K}+/ \mathrm{K}$ -

Pretzelosity on pi+/pi-

SSA: inclusive hadron

SSA: inclusive electron DIS

SSA: inclusive electron (Quasi)Elastic

- Planned SoLID program with JLab12

Precision 4-d mapping of TMD asymmetries

- EIC@HIAF opens up a new window

Exciting new opportunities

Precision experimental data + development in TMD theory (QCD evolution...)+...

$>$ lead to breakthrough in understanding QCD? 